\title{
A Regularized Profile Likelihood Approach to Covariance Matrix Estimation
}

\author{
Samprit Banerjee ${ }^{\mathrm{a}}$, Stefano Monni ${ }^{\mathrm{b}}$, Martin T. Wells ${ }^{\mathrm{c}}$ \\ ${ }^{a}$ Cornell University \\ ${ }^{b}$ American University of Beirut \\ ${ }^{c}$ Cornell University
}

\begin{abstract}
Two new orthogonally equivariant estimators of the covariance matrix are proposed. The estimates of the population eigenvalues are isotonized maximum likelihood estimates of the modified profile likelihood obtained from the Wishart distribution, in one case, and of a penalized form of such a likelihood function, in the other, with a penalty that constrains the trace of the sample covariance matrix. Properties of these estimators are studied and numerical risk comparisons with six other well-known estimators are presented to demonstrate the robustness of the proposed estimators for various real and simulated covariance structures.
\end{abstract}

Keywords: covariance matrix, eigenvalues, isotonic regression, Laplace approximation, modified profile likelihood.

\section{Introduction}

We observe $N$ independently distributed $p$-dimensional random vectors $x_{i}^{T}$, $i=1, \ldots, N$, drawn from a multivariate normal distribution with zero mean and unknown covariance matrix $\Sigma$, with $N>p$. This paper is concerned with the problem of estimating $\Sigma$ from the data $X=\left(x_{1}, \ldots x_{N}\right)^{T}$ (an $N \times p$ matrix). The usual unbiased estimator of the covariance matrix is the sample covariance matrix $S=\sum_{i}^{N}\left(x_{i}-\bar{x}_{i}\right)\left(x_{i}-\bar{x}_{i}\right)^{T} /(N-1)$, with $\bar{x}_{i}$ being the $p$-vector of the sample mean. Let $S=H L H^{T}$ and $\Sigma=O \Lambda O^{T}$ be the spectral decompositions of $S$ and $\Sigma$, so that $H$ and $O$ are the matrices of an orthonormal basis of eigenvectors (elements of the real orthogonal group $O(p))$ and $L=\operatorname{diag}(l)$ and $\Lambda=\operatorname{diag}(\lambda)$ are the diagonal matrices of the eigenvalues $l=\left(l_{1}, \ldots, l_{p}\right)$ and $\lambda=\left(\lambda_{1}, \ldots, \lambda_{p}\right)$, respectively. Throughout this paper we will assume that the sample and population eigenvalues are ordered, i.e. $l_{1}>l_{2}>\ldots>l_{p}>0$ and $\lambda_{1}>\lambda_{2}>\ldots>\lambda_{p}>0$.

Email addresses: sab2028@med.cornell.edu (Samprit Banerjee), sm150@aub.edu.lb (Stefano Monni), mtw1@cornell.edu (Martin T. Wells)

Preprint submitted to Elsevier

June 10, 2016 
The sample covariance matrix, $S$, follows a $p$-dimensional Wishart distribution with $n=N-1$ degrees of freedom and expectation $\Sigma$. One disadvantage of $S$ is that its eigenspectrum is more spread out than the population spectrum unless $\gamma=p / N$ is negligible, i.e. the largest eigenvalue of $S$ has a positive (upward) bias and the smallest has a negative (downward) bias. This has long been known and can be proven in a number of ways. In a language that will often be employed in this paper we can say that it is a direct consequence of the fact that the vector $E(l)=\left(E\left(l_{1}\right), \ldots, E\left(l_{p}\right)\right)$ of the expectations of the eigenvalues of $S$ majorizes the vector $\lambda$ of the eigenvalues of $\Sigma$. In other words, $\sum_{i=1}^{J} E\left(l_{i}\right) \geq \sum_{i=1}^{J} \lambda_{i}$ for any $J=1, \ldots, p-1$, and $\sum_{i=1}^{p} E\left(l_{i}\right)=\sum_{i=1}^{p} \lambda_{i}$. This is a corollary of a more general theorem for random Hermitian matrices due to Cacoullos and Olkin [7, 21].

Many studies have been dedicated to finding improved covariance matrix estimators that are less affected by the sample eigenvalue dispersion issue or that are better than $S$ in terms of risk. One approach looks for estimators that are equivariant under a group of transformations that acts on the sample space. Generally, the adequacy of the resulting estimator is studied with respect to loss functions that are invariant under the same group so that the entire decision problem is indeed invariant. Another approach imposes constraints on the sample covariance matrix in the form of regularization (for a review see $[27])$. In this article, we follow the first approach and consider estimators that are functions of $S$ and are equivariant under the orthogonal group $O(p)$. Namely, when the sample space is acted on by the orthogonal group according to $X^{T} \mapsto$ $O X^{T}$, so that $S \mapsto O S O^{T}, \Sigma \mapsto O \Sigma O^{T}$, we require the estimators to transform as $\phi(S) \mapsto \phi\left(O S O^{T}\right)=O \phi(S) O^{T}$. Orthogonally equivariant estimators $\phi(S)$ are of the form $\hat{\Sigma}=\phi\left(H L H^{T}\right)=H \hat{\Lambda} H^{T}$, with $\hat{\Lambda}=\phi(L)=\operatorname{diag}(\hat{\lambda})$ the matrix of the estimators $\hat{\lambda}=\left(\hat{\lambda}_{1}, \ldots, \hat{\lambda}_{p}\right)$ of the population eigenvalues $\lambda=\left(\lambda_{1}, \ldots, \lambda_{p}\right)$. Hence, equivariant estimators differ from each other only in how they estimate the population eigenvalues. Geometrically, the level set $Q_{\hat{\Sigma}}(z)=\left\{z: z^{T} \hat{\Sigma} z=\right.$ $\left.c, c>0, z \in \mathbb{R}^{p}\right\}$, of the positive definite quadratic form of such an estimator $\hat{\Sigma}$ is an ellipsoid with the same principal axes as the level set of the quadratic form of $S$, but different lengths. Thus the level sets of the orthogonally invariant estimators are a reshaping of the sample covariance matrix ellipsoids but have the same orientation. One of the best (in terms of risk) orthogonally equivariant estimators was proposed by Stein [33, 34], who obtained $\hat{\lambda}$ by (approximately) minimizing the unbiased estimator of the risk under Stein's loss (denoted by $L S$ ) [32] (see Section 5 for definition). Problems with such estimator were remedied by adjusting the eigenvalue estimates so as to make them positive, and, via isotonic regression, ordered. We will henceforth refer to the resulting estimator as Stein's estimator. A similar estimator was also proposed by Haff in [10], where $\hat{\lambda}$ are obtained by minimizing the Bayes risk under $L S$ (and a second one under the quadratic loss, denoted by $L Q$, see Section 5). Other orthogonally equivariant estimators proposed in the literature include Dey and Srinivasan [9], Perron [25, 26], Takemura [35] (see also the review [24]), and more recently Ledoit and Wolf $[16,17,18,19]$. In [17] the latter authors introduce a non- 
linear shrinkage estimator, where eigenvalues are obtained by applying a nonlinear transformation to the sample eigenvalues. They show how such estimator provides improvements over $S$ and performs better than the linear shrinkage estimator [16] constructed by shrinking all sample eigenvalues with the same intensity toward their mean. The nonlinear shrinkage approach was further extended to the $p>N$ case in [19] and [18] where estimators were proposed that are asymptotically optimal in the class of orthogonally equivariant estimators under the Frobenius loss (see Section 5) and under LS, respectively.

Naturally, groups of transformations other than the orthogonal group can be considered. For general linear transformation (invertible matrices), the equivariant estimators are known to be proportional to $S$ [32]. Much consideration has also been given to transformations corresponding to lower triangular matrices with positive diagonal elements. The corresponding group $\mathcal{G}_{T}^{+}$acts transitively on the space $\mathcal{S}_{p}^{+}$of positive definite symmetric matrices with the implication that the risk of a $\mathcal{G}_{T}^{+}$-equivariant estimator under a $\mathcal{G}_{T}^{+}$-invariant loss is constant. For example, Stein $[14,32]$ obtained one such estimator, which has since been shown to be dominated (under Stein's loss) by the orthogonally equivariant Stein's estimator, among others. In addition, orthogonally equivariant estimators are generally more interesting because, contrary to the $\mathcal{G}_{T}^{+}$-equivariant ones, they do not depend on the coordinate system in the sample space, as a change of basis is achieved by elements of $O(p)$.

Symmetry is not the only principle that can be employed to constrain a problem. Among the more recent literature, banding or tapering the sample covariance matrix is used by Bickel and Levina [6] to define regularized estimators. Regularization via banding or tapering assumes the variables have a natural ordering and thus it is appropriate only for problems with a precise underlying structure. Bickel and Levina also employ hard thresholding to regularize the sample covariance matrix [5] and obtain an estimator which is equivariant under permutations (which form a subgroup of the orthogonal group) and thus does not require the data to be ordered. They show that both the banded and thresholded estimators are consistent in the operator norm as long as $(\log p) / N \rightarrow 0$. In this paper, however, we will focus on the finite sample properties of covariance matrix estimation, particularly in the high-dimensional setting, when $N$ and $p$ are both large but $p<N$.

The starting point of our construction is the joint distribution of the sample eigenvalues which is obtained by integrating out the eigenvectors with respect to the Haar measure of $O(p)$ [23]. In our setting, the parameter space, which is $\mathcal{S}_{p}^{+}$, coincides with the sample space, if we look at the data via their sufficient statistic $S$. The Wishart density $p(S \mid \Lambda, O)$ (with respect to the invariant measure $\left.\mu(d S)=d S /|S|^{(p+1) / 2}\right)$ is such that $p\left(G^{-1}(S) \mid \Lambda, O\right)=p(S \mid \Lambda, G O)$ with $G \in O(p)$, where the $O(p)$-action is defined as $G(A)=G A G^{T}, A \in \mathcal{S}_{p}^{+}$. It follows that the marginal density of the sample eigenvalues depends only on the population eigenvalues. The marginal likelihood $f(\Lambda \mid L)$ of the invariant parameter $\Lambda$ can also be written as an integral over the Haar measure of the parameter $O, \int_{O(p)} f(\Lambda, O \mid S)(d O)$. The complete form of this marginal likelihood is how- 
ever intractable. For this reason, we consider its Laplace approximation, given in eq. (2). This approximate likelihood is the modified profile likelihood ([4], [23], [28]). As eigenvalue estimators, we propose the isotonic regression of the $n^{-1}$-order maximizers of this profile likelihood. Isotonic regression is considered to guarantee the correct ordering. We show that these estimates are positive, so there is no need for an additional computational step to obtain positivity, as is necessary in the implementation of Stein's estimator. We then propose a penalized estimator, which is the isotonic regression of the maximizers of a penalized likelihood function, where the penalty can constrain the trace of the covariance matrix estimator to be a fraction of the trace of the sample covariance matrix. We investigate finite sample properties of this estimation procedure through risk comparisons with other estimators. Large sample properties (for fixed $p$ ) of the sample eigenvalues, eigenvectors and the sample covariance matrix are well established: they are consistent estimators of their population counterparts, and asymptotic distributions of sample eigenvalues and eigenvectors are normal distributions centered around their population eigenvalues and eigenvectors. The proposed estimators perform reasonably well under various loss functions and different covariance structures, which we see as important because assumptions on the population covariance structure are often difficult to justify when dealing with data. One limitation of our estimation procedure is that it works when $p<N$, because of the use of the Laplace approximation. A special treatment is required when we try to extend it to the $p>N$. Such an extension will be presented in a separate manuscript.

This paper is organized as follows. In Section 2 we review the marginal distribution of the sample roots, and the maximum likelihood estimates. In Section 3 we introduce our first estimator based on these maximum likelihood estimates and study its properties. In Section 4 we obtain our second estimator derived from the penalized likelihood. In Section 5 we compare the proposed estimators to others proposed in the literature, by computing the risk based on some orthogonally invariant losses for various real and simulated covariance structures. In Section 6, we consider their use in a classification problem with linear discriminant analysis applied to a breast cancer dataset where the goal is to predict chemotherapy response from a multigene predictor. Section 7 contains some concluding remarks.

\section{Marginal distribution of the sample eigenvalues and Anderson's solution}

To fix notation, let $S=\sum_{i}^{N}\left(x_{i}-\bar{x}_{i}\right)\left(x_{i}-\bar{x}_{i}\right)^{T} / n$ be the sample covariance matrix for $N=n+1$ random samples from a Gaussian distribution with mean $\mu=0$ and covariance matrix $\Sigma$ and $S=H L H^{T}$ be its spectral decomposition, with the eigenvalue ordering previously specified. It is common to work with a version of $S$ that does not subtract the mean, however, we prefer to remove the mean so that we work with Wishart distribution with $n$ degrees of freedom $\mathcal{W}_{p}(n, \Sigma / n)$. When $n>p-1$, the joint marginal distribution of the $p$ eigenvalues 
is known to be

$$
f\left(l_{1}, \ldots, l_{p} \mid \Sigma\right)=c(n, p) \prod_{i} l_{i}^{\frac{n-p-1}{2}} \prod_{i<j}\left(l_{i}-l_{j}\right)|\Sigma|^{-n / 2} F_{0}^{(p)}\left(-n L / 2, \Sigma^{-1}\right),
$$

with

$$
F_{0}^{(p)}\left(-n L / 2, \Sigma^{-1}\right)=\int_{O(p)} \operatorname{etr}\left(-\frac{n}{2} \Sigma^{-1} H L H^{T}\right)(d H)
$$

(see for example [23]). The integral in (1) can be expressed in terms of zonal polynomials $[12,13]$. When $n$ is large, a Laplace approximation to this integral was given by Anderson [1]. The resulting modified profile likelihood function is

$$
\psi(\lambda \mid l)=\prod_{i} \exp \left(-\frac{n}{2} \frac{l_{i}}{\lambda_{i}}\right) \lambda_{i}^{-n / 2} \cdot \prod_{i<j}\left(\frac{\lambda_{i} \lambda_{j}}{\lambda_{i}-\lambda_{j}}\right)^{1 / 2},
$$

where the first product term is the profile likelihood function and the second product term is the $\lambda$-dependent terms of the inverse of the square root of the determinant of the observed information matrix for $O$ evaluated for fixed $\lambda$ at the partial maximum likelihood estimate $\hat{O}_{\lambda}=H$ (which coincides with the maximum likelihood estimate $\hat{O}$ ). The central idea of Anderson's proof is to convert the Haar measure to a Lebesgue measure and carry out Laplace expansion around $\sum l_{i} / \lambda_{i}$. Anderson [1] also gives an expression for the solutions $\breve{\lambda}_{i}^{m l e}$ of the maximum profile likelihood equations,

$$
l_{i}=\lambda_{i}+\frac{\lambda_{i}}{n} \sum_{j \in I_{-i}} \frac{\lambda_{j}}{\lambda_{i}-\lambda_{j}}
$$

where $I_{-i}=\{1, \ldots, i-1, i+1, \ldots, p\}$. It can be shown

$$
\breve{\lambda}_{i}^{m l e}=l_{i}-\frac{l_{i}}{n} \sum_{j \in I_{-i}} \frac{l_{j}}{l_{i}-l_{j}}+O\left(n^{-2}\right) \equiv \tilde{\lambda}_{i}+O\left(n^{-2}\right) .
$$

Although tractable expressions for the exact moments of the eigenvalues of $S$ are unknown, Lawley [15] obtains asymptotic expansions of the same

$$
E\left(l_{i}\right)=\lambda_{i}+\frac{\lambda_{i}}{n} \sum_{j \in I_{-i}} \frac{\lambda_{j}}{\lambda_{i}-\lambda_{j}}+O\left(n^{-2}\right) .
$$

This shows that the sample eigenvalues have a bias of $O\left(n^{-1}\right)$, which is positive for $l_{1}$ (since $\lambda_{1}>\lambda_{j}$ for all $j \neq 1$ ) and negative for $l_{p}$ (since $\lambda_{p}<\lambda_{j}$ for all $j \neq p)$. Using Lawley's expression for moments the expected value of $\breve{\lambda}^{\text {mle }}$ can be easily obtained:

$$
E\left(\breve{\lambda}_{i}^{m l e}\right)=\lambda_{i}+O\left(n^{-2}\right)
$$

which demonstrates that the bias reduces to $O\left(n^{-2}\right)$. This reduction in bias could be attributed to the fact that these eigenvalue estimates utilize information 
from all other eigenvalues with the adjacent ones having the most effect; this type of shrinkage is similar to the pattern in the Stein's estimate. Also, for large $n$ the bias vanishes and it is known that the $l_{i}$ 's are consistent estimators of the corresponding $\lambda_{i}$ 's $[2]$.

The starting point of our analysis is the leading terms of Anderson's solution:

$$
\tilde{\lambda}_{i}=l_{i}-\frac{l_{i}}{n} \sum_{j \in I_{-i}} \frac{l_{j}}{l_{i}-l_{j}} \equiv l_{i}+\frac{l_{i}}{n} A_{i} .
$$

It is unreasonable to use the vector $\tilde{\lambda}=\left(\tilde{\lambda}_{1}, \ldots, \tilde{\lambda}_{p}\right)$ as estimator of the eigenvalues $\lambda$ of $\Sigma$, because some components may be negative. This is so because $A_{i}$ is not positive for some $i$ (for example $A_{1}<0$ ). Furthermore, if all components were positive, they might be non-ordered, and it would then be preferable to consider the estimator that resulted after some re-ordering procedure, as shown in [31]. The next section is devoted to some modifications of Anderson's solution which give rise to well-defined estimators.

\section{The Isotonized Maximum Modified (Profile) Likelihood Estimator (IMMLE)}

As estimators for the eigenvalues $\lambda$, we propose the isotonic regression of $\tilde{\lambda}=\left(\tilde{\lambda}_{1}, \ldots, \tilde{\lambda}_{p}\right)$. Namely, we look for $\hat{\lambda}=\left(\hat{\lambda}_{1}, \cdots, \hat{\lambda}_{p}\right)$ which minimize

$$
\sum_{i=1}^{p}\left(\tilde{\lambda}_{i}-\hat{\lambda}_{i}\right)^{2}
$$

subject to the ordering constraint $\hat{\lambda}_{1} \geq \hat{\lambda}_{2} \geq \ldots \geq \hat{\lambda}_{p}$. In the equivalent min-max formulation [3] these solutions are given by

$$
\hat{\lambda}_{i}=\min _{s \leq i} \max _{t \geq i} \frac{\sum_{r=s}^{t} \tilde{\lambda}_{r}}{\sum_{r=s}^{t} 1} .
$$

The pool adjacent violators algorithm [3] can be used to solve (5). We call the isotonic regression $\hat{\lambda}$ the Isotonized Maximum Modified (profile) Likelihood Estimator (IMMLE) of $\lambda$. Similarly, we refer to the corresponding orthogonally equivariant estimator $\hat{\Sigma}=H \hat{\Lambda} H^{T}$, with $\hat{\Lambda}=\operatorname{diag}(\hat{\lambda})$, as the IMMLE of $\Sigma$. While in general the isotonic regression estimates may be negative, in our case we are able to show that they are positive. This and other properties of the eigenvalue IMMLE are summarized in the following

Proposition 1. (The properties of the IMMLE) The IMMLE $\hat{\lambda}$ has the following properties

a) $\hat{\lambda}_{1} \geq \hat{\lambda}_{2} \geq \ldots \geq \hat{\lambda}_{p}>0$

b) $\sum_{i=1}^{p} \hat{\lambda}_{i}=\sum_{i=1}^{p} l_{i}$ 
c) $\sum_{i=1}^{J} \hat{\lambda}_{i}<\sum_{i=1}^{J} l_{i}, \quad J=1, \ldots, p-1$.

The proof of Proposition 1 is given in Appendix A.

Remark 1 . Since the components of $\hat{\lambda}$ are ordered and positive, the covariance IMMLE is a well-defined estimator of a positive definite matrix.

Remark 2. Given that the components of $\hat{\lambda}$ and $l$ are in non-increasing order, properties b) and c) can be summarized with the statement that the vector of the eigenvalues of the IMMLE is (strictly) majorized by the vector of the sample eigenvalues [21]. In mathematical notation, we write $\hat{\lambda} \prec l$. In particular, $l_{1}>\hat{\lambda}_{i}>l_{p}, i=1, \cdots, p$ : the spectrum of the IMMLE is shrunk as compared to that of $S$, i.e. the largest and smallest eigenvalues of the IMMLE are closer to each other than those of $S$. As observed in the Introduction, $\lambda \prec E(l)=$ $\left(E\left(l_{1}\right), \ldots, E\left(l_{p}\right)\right)$ so that the property of a population eigenvalue estimator to be majorized by the sample eigenvalues is a necessary (albeit not sufficient) condition for it to improve the sample estimator, if we use majorization as a measure of adequacy of an estimator.

Remark 3. The trace of the IMMLE is equal to the trace of $S$. This seems to be a desirable property since $E(\operatorname{tr} S)=\operatorname{tr} \Sigma$. We will comment on this point later, when we introduce an estimator that forgoes this property.

Remark 4. More generally, for all elementary symmetric functions, $S_{k}(x)=$ $\sum_{i_{1}<\ldots<i_{k}} x_{i_{1}} \cdots x_{i_{k}}, E\left(S_{k}(l)\right)=S_{k}(\lambda) \prod_{i=1}^{k}\left(1-\frac{1}{n}(i-1)\right) \leq S_{k}(\lambda)$, (see, e.g., $[23])$, so that there is an underestimation of the symmetric functions associated with $\Sigma$ when $k>1$. In our case, majorization implies $S_{k}(l) \leq S_{k}(\hat{\lambda})$ with strict inequality when $k>1$, because then $S_{k}(x)$ is strictly Schur-concave on $\mathbb{R}_{>0}^{p}=\left\{x=\left(x_{1}, \ldots, x_{p}\right) \in \mathbb{R}^{p} \mid x_{i}>0\right\}$ and $\hat{\lambda}$ is not a permutation of $l$ (see e.g. [21]). In particular, $E(\operatorname{det} S)<\operatorname{det} \Sigma$ and $\operatorname{det} S<\operatorname{det} \hat{\Sigma}$.

Remark 5. Under scale transformations, $l_{i} \rightarrow a l_{i}$, for all $i, a>0$, the IMMLE changes accordingly: $\hat{\lambda}_{i} \rightarrow a \hat{\lambda}_{i}$, for all $i$. This equivariance under scaling of the eigenvalue estimates clearly extends to the covariance matrix estimator: under $S \rightarrow a S, \hat{\Sigma} \rightarrow a \hat{\Sigma}$.

Since Anderson's solution (3) is an approximate solution that ignores terms of order $n^{-2}$ and higher, it is natural that one might want to consider a higher order correction. It can be shown that the solution of the MLE equations that retains the next order term is

$$
\tilde{\tilde{\lambda}}_{i}=l_{i}+\frac{1}{n} l_{i} A_{i}+\frac{1}{n^{2}} \sum_{k \in I_{-i}} \frac{l_{i} l_{k}}{\left(l_{i}-l_{k}\right)^{2}}\left(l_{k} A_{i}-l_{i} A_{k}\right) .
$$

Even in this case, it is best to consider the isotonic regression of this solution, which can be referred to as the second-order IMMLE. Our simulation studies did not show, however, consistent improvements of this higher-order estimator over the IMMLE. 


\section{The Isotonized Maximum Penalized (Modified Profile) Likelihood Estimator (IMPLE)}

As we have previously noticed, one of the features of the IMMLE is that its trace is equal to that of $S$. While this is a desirable property as the expectation of $\operatorname{tr} S$ is the trace of the population covariance matrix $\Sigma$, we have empirically observed that many estimators that perform better than $S$ under most loss functions have traces that are smaller than $S$. Thus we now consider a modified estimator, obtained by maximizing a penalized likelihood in which the penalty controls the trace of the estimator to differ from and in particular be smaller than that of $S$, for finite $n$. Namely, we add to the logarithm of the modified profile likelihood (2) the penalty function

$$
P(\lambda, \kappa)=\sum_{i=1}^{p} \lambda_{i}-\sum_{i=1}^{p} l_{i}\left(1-\frac{\kappa}{n}\right),
$$

where $\kappa$ is a constant, $\kappa<n$, which, if positive, gives rise to the shrinkage of the trace of the estimator, with larger values increasing the shrinkage. This penalty is consistent with the fact that in the large $n$ limit we want to recover the sample eigenvalues, which are consistent estimators of $\lambda$. The ML equations derived from this penalized log-likelihood

$$
\log \psi(\lambda, \kappa \mid l)=\log \psi(\lambda \mid l)+\mu\left(\sum_{i=1}^{p} \lambda_{i}-\sum_{i=1}^{p} l_{i}\left(1-\frac{\kappa}{n}\right)\right)
$$

are then:

$$
\begin{aligned}
l_{i} & =\lambda_{i}+\frac{\lambda_{i}}{n} \sum_{j \in I_{-i}} \frac{\lambda_{j}}{\lambda_{i}-\lambda_{j}}-\lambda_{i}^{2} \frac{2}{n} \mu, \\
\sum_{i=1}^{p} \lambda_{i} & =\sum_{i=1}^{p} l_{i}\left(1-\frac{\kappa}{n}\right) .
\end{aligned}
$$

We have the following:

Proposition 2. (Solutions to the penalized ML equations) The solutions to the penalized $M L$ equations are

$$
\tilde{\lambda}_{i}^{\kappa}=l_{i}+\frac{l_{i}}{n} A_{i}-\frac{\kappa}{n} \frac{\operatorname{tr}(S)}{\operatorname{tr}\left(S^{2}\right)} l_{i}^{2}=\tilde{\lambda}_{i}^{0}-\frac{\kappa}{n} \frac{\operatorname{tr}(S)}{\operatorname{tr}\left(S^{2}\right)} l_{i}^{2} .
$$

Here $\tilde{\lambda}_{i}^{0}$ is the leading terms (3) of Anderson's solution. The details of the proof of Proposition 2 are given in Appendix C. In this case too, the MLE estimator $\tilde{\lambda}^{\kappa}=\left(\tilde{\lambda}_{1}^{\kappa}, \cdots, \tilde{\lambda}_{p}^{\kappa}\right)$ is not guaranteed to be positive or ordered. Thus, we consider its isotonic regression $\hat{\lambda}^{\kappa}=\left(\hat{\lambda}_{1}^{\kappa}, \ldots, \hat{\lambda}_{p}^{\kappa}\right)$ as our estimator, which we name the IMPLE, as it is the Isotonic regression of the Maximizers of the Penalized Likelihood. The IMPLE reduces to the IMMLE when $\kappa=0$, thus from now $\hat{\lambda}^{0}$ is used to denote the latter. We have 
Proposition 3. (The properties of the IMPLE) The IMPLE $\hat{\lambda}^{\kappa}$ has the following properties

a) $\hat{\lambda}_{1}^{\kappa} \geq \hat{\lambda}_{2}^{\kappa} \geq \ldots \geq \hat{\lambda}_{p}^{\kappa}>0$ for all $\kappa$

b) $\hat{\lambda}^{\kappa}<\hat{\lambda}^{0}$ (that is, $\hat{\lambda}_{i}^{\kappa}<\hat{\lambda}_{i}^{0}, i=1, \ldots, p$ ) when $\kappa>0$.

The proof of Proposition 3 is given in Appendix A.

Remark 6. Property a) implies that the eigenvalue IMPLE is a well-defined estimator of the population eigenspectrum with ordered and positive eigenvalue estimates. Accordingly, the covariance IMPLE is a positive definite estimator of the covariance matrix.

Remark 7. Property b) implies that when $\kappa>0 \hat{\lambda}^{\kappa}$ is weakly sub-majorized by $\hat{\lambda}^{0}$ (and hence by $l$ ). Following the notation of [21], we write $\hat{\lambda}^{\kappa} \prec_{w} \hat{\lambda}^{0}$.

Remark 8. $\operatorname{tr} \hat{\Sigma}^{\kappa}=\left(1-\frac{\kappa}{n}\right) \operatorname{tr} S$ because isotonization preserves the sum.

Remark 9. When $\kappa>0$, det $\hat{\Sigma}^{\kappa}<\operatorname{det} \hat{\Sigma}^{0}$ from b). However $\operatorname{det} \hat{\Sigma}^{\kappa}$ can be larger than, or smaller than, or equal to $\operatorname{det} S$.

Remark 10. The IMPLE is equivariant under scale transformations.

In this case as well we can consider higher-order solutions of the ML equations (8). At order $n^{-2}$, we obtain the solutions

$$
\begin{aligned}
\tilde{\tilde{\lambda}}_{i}^{\kappa}= & l_{i}+\frac{1}{n} l_{i} A_{i}+\frac{1}{n^{2}} \sum_{k \in I_{-i}} \frac{l_{i} l_{k}}{\left(l_{i}-l_{k}\right)^{2}}\left(l_{k} A_{i}-l_{i} A_{k}\right) \\
& -\frac{r_{\kappa}}{n} l_{i}^{2}+2 \frac{r_{\kappa}^{2}}{n^{2}} l_{i}^{3}-2 \frac{r_{\kappa}}{n^{2}} l_{i}^{2} A_{i},
\end{aligned}
$$

where $r_{\kappa}=\kappa \operatorname{tr}(S) / \operatorname{tr}\left(S^{2}\right)$, and we can consider their isotonic regressions as eigenvalue estimators (the second-order IMPLE). Risk computations do not seem to indicate that the second-order IMPLE is generally better than the IMPLE.

\subsection{Selecting $\kappa$}

The IMPLE $\hat{\lambda}^{\kappa}$ depends on a parameter $\kappa$, which needs to be chosen. For the trace of the estimator to be positive, it must be $\kappa<n$. When $\kappa>0$ the trace of the IMPLE is less than $\operatorname{tr} S$, and when $\kappa<0$ the trace is expanded. There is nothing a priori that should prevent us from letting $\kappa$ be negative. Indeed, even when $\kappa<0$, the IMPLE is a well-defined estimator for a positive definite matrix. From our simulation experiments we observe however that it suffices to restrict $\kappa$ to be in the range $[0, n)$. The shrinkage parameter $\kappa$ can be chosen by minimizing the risk under some loss function $L(\hat{\Sigma}, \Sigma)$ :

$$
R(\hat{\Sigma}, \Sigma)=E(L(\hat{\Sigma}, \Sigma)),
$$

where the expectation is over the data distribution. When $\hat{\Sigma}=\hat{\Sigma}^{\kappa}$, the risk can be seen as a function of $\kappa$. The "oracle" $\kappa$ is then $\kappa^{\prime}=\underset{\kappa}{\operatorname{argmin}} R(\kappa)$. However, 
in practice the true population matrix $\Sigma$ is never known and we thus resort to a resampling scheme, to which we refer as the selective-bootstrap. From the given data $X$, an $N \times p$ matrix, we obtain a random matrix $X_{b}$ by first sampling $p+1$ rows of $X$ without replacement, and then sampling other $N-p-1$ rows with replacement. This variant of the bootstrap sampling scheme guarantees that the corresponding sample covariance matrix $S_{b}$ (sufficient statistic) has full rank and is invertible. We then construct the IMPLE $\hat{\Sigma}_{b}^{\kappa}$ for a given $\kappa$, and compute the loss $L\left(\hat{\Sigma}_{b}^{\kappa}, \bar{\Sigma}\right)$ with respect to a reference estimator $\bar{\Sigma}$. We repeat the procedure for values of $\kappa$ belonging to a pre-arranged set $I_{\kappa}$ contained in the interval $[0, n-1]$ of the real line, for example the set of integers $\{0, \ldots, n-1\}$. We then iterate this procedure $B$ times, estimate the risk as follows:

$$
\hat{R}(\kappa)=\frac{1}{B} \sum_{b=1}^{B} L\left(\hat{\Sigma}_{b}^{\kappa}, \bar{\Sigma}\right)
$$

and choose for $\kappa$

$$
\hat{\kappa}=\underset{\kappa \in I_{\kappa}}{\operatorname{argmin}} \hat{R}(\kappa) .
$$

If $\hat{\kappa}=0$ then one can expand $I_{\kappa}$ to include $\kappa<0$ in order to find the minimum of the risk function. The above procedure requires us to specify a reference estimator $\bar{\Sigma}$ and a loss function $L(\cdot)$. We propose using the sample covariance matrix $S$ (of $X$ ) or the IMMLE $\left(\hat{\Sigma}^{0}\right)$ as the reference estimator and we refer to these procedures as BS and BIMMLE respectively.

For constructing Figure 1, we computed a Monte Carlo estimate of the "oracle" risk (9) by averaging over 100 data sets and computed $\hat{R}(\kappa)$ from a single dataset by the resampling scheme described above. This procedure was repeated for a variety of loss functions, different covariance structures, and various values of $p$ and $N$ (see Section 5.3), although only part of the analysis is presented here. Figure 1 displays the risk function when the loss is the absolute difference of the eigenvalues ( $L 1$, defined in Section 5.2), when the population covariance matrix has widely spaced eigenvalues, $\Sigma_{1}$, or one large eigenvalue, $\Sigma_{2},\left(\Sigma_{1}\right.$ and $\Sigma_{2}$ are properly defined in Section 5.3$)$ when $p=(10,20,50,100)$ and $p / N=\gamma=0.2,0.8$ for each $p$. Figure 1 shows that the selective-bootstrap procedure with either choice of $\bar{\Sigma}$, BS or BIMMLE, is adequate for choosing $\kappa$. Our simulations suggest that there is no great need of considering as $I_{\kappa}$ a finer grid than a sequence of integers.

We also considered two other sampling schemes: $K$-fold cross validation (in which the testing and training set are determined as usual) and regular bootstrap in which the training set $X_{b}$ is obtained by randomly sampling with replacement $N$ rows of $X$. Our extensive simulations suggest the proposed selective bootstrap method to be the best.

\subsection{Some observations on a different class of penalized estimators}

The IMMLE is obtained by minimizing eq. (4), that is, the square of the Euclidean distance ( $L_{2}$ loss) from Anderson's solution, under the ordering constraint, and thus it can be seen as a standard convex optimization problem. On 

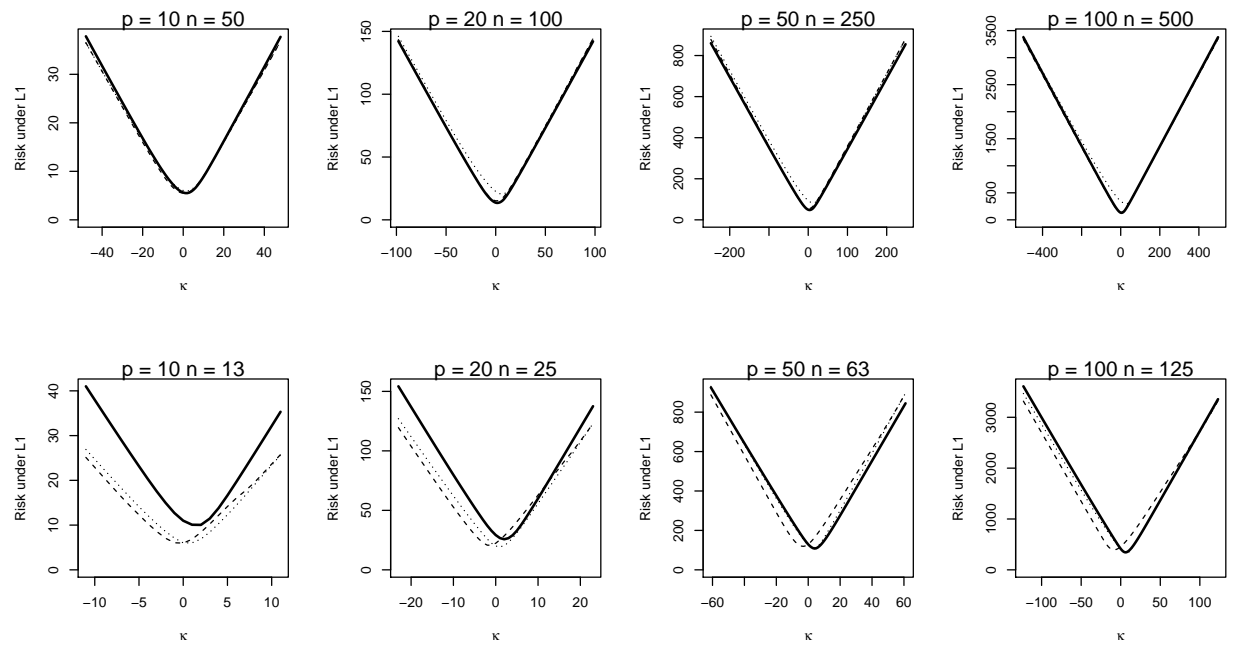

$$
\text { - } \text { oracle } \ldots \text { BS } \quad \text { B....... BIMMLE } \mid \quad \Sigma_{1}
$$
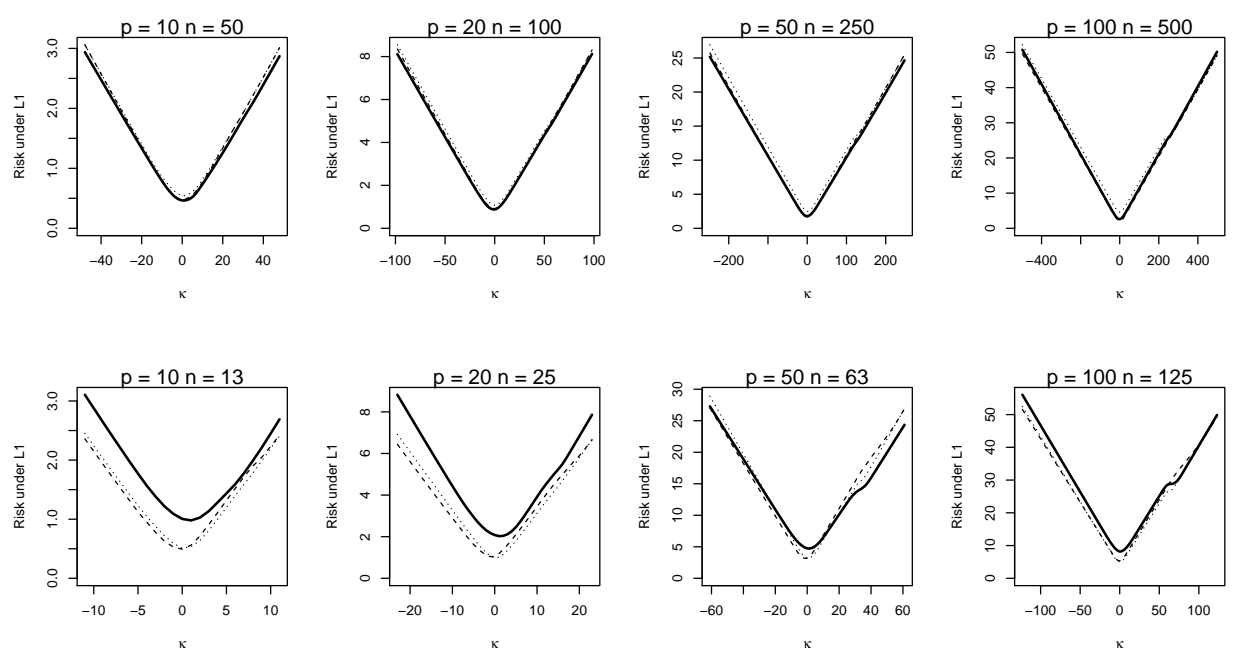

$\mid$ - oracle $\ldots$. BS $\quad$ B..... BIMMLE $\mid \quad \Sigma_{2}$

Figure 1: True (oracle) and estimated (BS and BIMMLE) risk as a function of $\kappa$. BS and BIMMLE correspond to the procedure of estimating risk via selective-bootstrap with $\bar{\Sigma}$ chosen to be $S$ and the IMMLE respectively. $\Sigma_{1}$ and $\Sigma_{2}$ are two different covariance matrices defined in Section 5.3. 
the other hand, the IMPLE is obtained by maximizing a penalized log-likelihood with a penalty term (6) that constrains the sum of the solutions. It is thus natural to consider a new class of estimators where the constraint $P()=$.0 , ordering and positivity are all enforced when minimizing the $L_{2}$ loss. This problem too is solvable, as it is a standard convex constrained optimization problem. Indeed, we did consider this problem and we obtained numerical solutions, but our simulations indicated that the IMPLE was generally a superior estimator. Another related optimization problem we studied consisted in adding a convex penalty term directly to the $L_{2}$ loss (4) and then minimizing the corresponding convex function under positivity and ordering constraints. Specifically, we dealt with the case where an entropy penalty was added to the $L_{2}$ function. In this case as well, no significant improvement was found.

\section{Risk Comparisons}

\subsection{Risk comparison with $S$}

In general, analytic computations of the risk for our estimators are out of reach without imposing the restrictive assumption that $S$ and $\Sigma$ have identical eigenvectors. However, in some special cases (see Appendix B for details), we are able to compare analytically $S$ and the IMMLE $\hat{\Sigma}^{0}$. When $\Sigma=c I$, we show that the IMMLE has lower risk than $S$ under four different loss functions. Namely,

Proposition 4. If $\Sigma=c I$ and under any of the four losses $L 1, L 2, L Q, L S$ (see 5.2 for definition):

$$
R\left(\hat{\Sigma}^{0}, \Sigma\right)-R(S, \Sigma) \leq 0
$$

with strict inequality except for $L 1$.

Using Stein's Unbiased Risk Estimator (SURE) [34], we can also show risk dominance of the IMMLE over $S$ with respect to Stein's loss $L S$, under certain assumptions, in two extreme cases: a) when the IMMLE eigenspectrum has maximum degeneracy, i.e., $\hat{\lambda}_{i}=\frac{\sum_{i=1}^{p} l_{i}}{p}$, for all $i$; and b) when the IMMLE eigenspectrum has no degeneracy, i.e. $\hat{\lambda}_{i}=\tilde{\lambda}_{i}$, for all $i$. The latter case arises when Anderson's solutions are ordered and positive and thus no isotonization is required.

\subsection{Numerical risk comparisons with other estimators}

Since analytical comparisons are generally not tractable we resort to Monte Carlo estimates of risk for a more detailed comparison. We want to compare our estimators, the IMMLE and the IMPLE, with six others: the sample covariance matrix, Stein's estimator, Ledoit and Wolf's nonlinear shrinkage estimator that is asymptotically optimal under Frobenius loss and that which is asymptotically optimal under Stein's loss, Bickel and Levina's banded and threshold estimators. We notice that we took into account the fact that some other authors employed 
different definitions of the sample covariance matrix. The Stein's estimator we consider for the comparison study is the orthogonally equivariant estimator that minimizes (approximately) the unbiased estimator of risk under the Stein's loss function $L S$. The details of the implementation can be found in [34] and [20]. Ledoit and Wolf's nonlinear shrinkage estimators are of the form $H \hat{D} H^{T}$, and thus orthogonally equivariant, with $\hat{D}=\operatorname{diag}\left(\hat{\varphi}^{*}\left(l_{1}\right), \ldots \hat{\varphi}^{*}\left(l_{p}\right)\right)$. The function $\hat{\varphi}^{*}$ is the nonlinear function responsible for shrinking the sample eigenvalues. Its form depends on which loss function is asymptotically minimized [18]. We refer the reader to Section 3.2 of [19] for the specific form of $\hat{\varphi} *$ when the loss is Frobenius and to Sections 5 and 6 of [18] for its explicit form when the loss is Stein's. The banded estimator of Bickel and Levina [6] is constructed by shrinking to 0 the elements of the sample covariance matrix that are outside a band of width $k$ off the diagonal. Namely,

$$
B_{k}(S)=\left[s_{i j} \mathbf{1}(|i-j| \leq k)\right]
$$

where $\mathbf{1}_{i j}=1$ if $|i-j| \leq k$ and 0 otherwise. The banding parameter $k,(0 \leq k<$ $p$ ) is chosen by a resampling scheme described in [6]. The threshold estimator [5] is similarly defined, where the regularization of $S$ is achieved shrinking its elements that are below a threshold $t$ to 0 :

$$
T_{t}(S)=\left[s_{i j} \mathbf{1}\left(\left|s_{i j}\right| \geq t\right)\right]
$$

The threshold $t>0$ is chosen by a resampling scheme described in [5].

Estimators can be compared considering differences of the risk, defined in eq. (9). The following seven loss functions will be considered in this paper:

1. the quadratic loss $L Q(\hat{\Sigma}, \Sigma)=\operatorname{tr}\left(\hat{\Sigma} \Sigma^{-1}-I\right)^{2}$,

2. Stein's (entropy) loss $L S(\hat{\Sigma}, \Sigma)=\operatorname{tr}\left(\hat{\Sigma} \Sigma^{-1}-I\right)-\log \operatorname{det}\left(\hat{\Sigma} \Sigma^{-1}\right)$,

3. $L_{2}$ eigenvalue loss $L 2(\hat{\Sigma}, \Sigma)=\sum_{i=1}^{p}\left(\hat{\lambda}_{i}-\lambda_{i}\right)^{2} / p$

4. $L_{1}$ eigenvalue $\operatorname{loss} L 1(\hat{\Sigma}, \Sigma)=\sum_{i=1}^{p}\left|\hat{\lambda}_{i}-\lambda_{i}\right| / p$,

5. $L_{1}$ loss on the largest eigenvalue $E V 1(\hat{\Sigma}, \Sigma)=\left|\hat{\lambda}_{1}-\lambda_{1}\right|$,

6. $L_{1}$ loss on the smallest quartile of the eigenvalues $E V S(\hat{\Sigma}, \Sigma)=\sum_{i=\lceil 3 p / 4\rceil}^{p} \mid \hat{\lambda}_{i}-$ $\lambda_{i} \mid$

7. Frobenius loss $\operatorname{LF}(\hat{\Sigma}, \Sigma)=\|\hat{\Sigma}-\Sigma\|_{F}$, with $\|A\|_{F}^{2}=\operatorname{tr}\left(A A^{T}\right)$.

The first two losses are the most widely used in covariance matrix estimation, and were introduced in [30] and [32], respectively. The third and fourth are based on the $L_{2}$ and $L_{1}$ norms of the eigenvalue vectors. The next two losses $(E V 1, E V S)$ are considered here to investigate the extremes of the eigenvalue spectrum and allow us to assess the shrinking of the eigenspectrum. They can also have some practical implications. For example, EV1 tells us which estimator is best for principal components analysis. The last loss is based on the (standard) Frobenius norm. These loss functions are invariant under orthogonal transformations (the first two under general linear transformations in fact), thus the entire decision process at hand is invariant under the orthogonal group. 
Accordingly, without loss of generality, one can choose $\Sigma$ to be diagonal in the risk computations that involve orthogonally equivariant estimators. However, Bickel and Levina's estimators are not orthogonally equivariant, thus their risk may vary along the $O(p)$-orbits in the space $\mathcal{S}_{p}^{+}$of covariance matrices. Because of the way the banded estimator is defined, it may have an unfair advantage over orthogonally equivariant estimators in the assessment of risk when $\Sigma$ is diagonal. Thus, in three of our simulation designs for $\Sigma$, we employed a random orthogonal transform of a diagonal matrix with a specific eigenvalue structure. Finally we notice that, for the IMPLE, the best shrinkage parameter $\kappa$ was chosen by selective-bootstrap (described in Section 4.1) using the first four loss functions ( $L Q, L S, L 1$ and $L 2)$.

\subsection{Simulation Design}

We have considered the following five different covariance structures, four artificially constructed and one obtained from real stock market data:

1. $\Sigma_{1}=G \Lambda G^{T}$ where $\Lambda=\operatorname{diag}\left(p^{2}, \cdots, 2^{2}, 1^{2}\right)$ and $G \in O(p)$;

2. $\Sigma_{2}=G \Lambda G^{T}$ where $\Lambda=\operatorname{diag}\left(\lambda_{(1)}^{*}, \lambda_{(2)}, \cdots, \lambda_{(p)}\right), \lambda_{i} \sim U(1, p / 2), \lambda_{(i)}$ are the ordered $\lambda_{i}$ 's, $\lambda_{(1)}^{*}=2 \lambda_{(1)}$ and $G \in O(p)$, with $U(a, b)$ being the uniform distribution over the $[a, b]$ interval;

3. $\Sigma_{3}=A R(1)$, the first-order autoregressive covariance matrix, where $\sigma_{i j}=$ $4 \times 0.7^{|i-j|}$ for $i \neq j$ and $\sigma_{i i}=4^{2}$ for $i=1, \cdots, p$;

4. $\Sigma_{4}=G \Lambda G^{T}$ where $\Lambda=\operatorname{diag}(2 p, p, 1, \cdots, 1)$ and $G \in O(p)$.

5. $\Sigma_{N 100}$, the sample covariance matrix of the daily returns of the $p=107$ securities that compose the NASDAQ-100 index recorded on the 167 trading days from March 6, 2014 to July 7, 2015. See [8] for more details.

We think these choices are representative of typical applications. Indeed, $\Sigma_{4}$ represents a spiked covariance structure, $\Sigma_{3}$ is a time series example, $\Sigma_{2}$ represents the typical principal components analysis covariance structure of one large eigenvalue and $\Sigma_{1}$ was generated to reflect the fact that the asymptotic expansions of marginal likelihood are valid when $\left(\lambda_{i}^{-1}-\lambda_{j}^{-1}\right)$ are widely spaced [22]. The NASDAQ covariance matrix is almost singular $\left(\log \operatorname{det} \Sigma_{N 100}=-1011.921\right)$ as many of its eigenvalues are very small and thus represents a numerically illconditioned situation.

For each $\Sigma_{i}(i=1,2,3,4)$ we consider $p=10,20,50,100$ and choose two values of $N$ corresponding to $p / N=\gamma=0.2,0.8$, for each $p$. We evaluate all seven loss functions, denoted henceforth by $L S, L Q, L 1, L 2, L F, E V 1$ and $E V S$, on all eight estimators denoted by IMMLE, IMPLE, $S$, Stein, LW1 (nonlinear shrinkage estimator that is optimal under Frobenius loss), LW2 (nonlinear shrinkage estimator optimal under Stein's loss), BL.band, BL.thresh (see Section 5.2). IMPLE denotes the best IMPLE (in terms of risk) among the four $\kappa$-determining methods based on four loss functions. For $\Sigma_{N 100}$, we consider two cases, $N=125(\gamma=0.856)$ and $N=250(\gamma=0.428)$, and compute all eight estimators. However, we consider the three matrix loss functions only $(L S, L Q$ and $L F$ ) because the eigenvalue based loss functions may not be meaningful in 
such a numerically ill-conditioned situation. Risk estimates are based on 1000 repetitions for each design.

\subsection{Simulation Results}

The Proportion Reduction in Integrated Average Loss (PRIAL) for an estimator $\hat{\Sigma}$ in relation to $S$ is computed and presented in Table 3, Table 4, Table 5 , Table 6 and Table 7 (of Appendix D) for $\Sigma$ equal to $\Sigma_{1}, \Sigma_{2}, \Sigma_{3}, \Sigma_{4}$ and $\Sigma_{N 100}$ respectively. PRIAL is defined as in [20] $\left(\sum_{i} L\left(S_{i}, \Sigma\right)-\sum_{i} L\left(\hat{\Sigma}_{i}, \Sigma\right)\right) / \sum_{i} L\left(S_{i}, \Sigma\right)$, where the sum is over all datasets and $L($.$) is a generic loss function. Figures 2,$ $3,4,5$ and 6 visually represent the PRIAL for a subset of loss functions for $\Sigma_{1}$, $\Sigma_{2}, \Sigma_{3}, \Sigma_{4}$ and $\Sigma_{N 100}$ respectively. Not all estimators are included in each panel of Figures 2, 3, 4 and 5. Negative PRIAL values are not included in the figures; as a result, estimators having consistently higher risk than $S$ are excluded. For $\Sigma_{1}$ (see Figure 2 and Table 3), the IMPLE (followed by the IMMLE) has good performance under all loss functions. Even when it is not the best, it is close to the best. BL.band performs well under some losses (e.g. $L F$ and $L 1$ ) while it is worse than $S$ for others (e.g. $L S$ and $L Q$ ). Similarly, Stein's estimator improves risk under $L S$ and $E V S$ but performs poorly under $L 1$ and $L 2$. For the covariance structure $\Sigma_{2}$, which has one large eigenvalue, all estimators (including $S$ ), except Stein's and LW2, perform well in estimating the largest eigenvalue (see Figure 3 and Table 4) under EV1 loss. Similar to $\Sigma_{1}$, the IMPLE (followed by the IMMLE) performs well under all loss functions. Under $L S$, Stein's is the best followed by LW2. This is not surprising as Stein's estimator minimizes the risk under $L S$ and LW2 is optimal under $L S$. For $\Sigma_{3}$ (see Figure 4 and Table $5)$, however, the IMMLE and the IMPLE are mostly the best in estimating the highest eigenvalue ( $E V 1$ loss) but LW1, LW2 and BL.band are slightly better with other loss functions. For the spiked covariance structure $\Sigma_{4}$, the IMPLE shows maximum risk reduction under $L F$ but moderate risk improvement under other loss functions. LW1 shows substantial risk improvement under $L S$, $L Q, L 1$ and $E V S$ while it performs moderately under $L 2$ and $E V 1$; LW2, on the other hand, shows substantial risk improvement under $L S, L Q$, and $E V S$ while it performs poorly under $L F, L 1$ and $E V 1$. Under $E V 1$, all estimators (including $S$ ) estimate the largest eigenvalue well except Stein's and LW2. For $\Sigma_{N 100}$ (see Figure 6 and Table 7 ) most estimators perform rather poorly and are worse than $S$ in most situations, however, the IMPLE is the only estimator which shows risk improvement for both sample sizes and all loss functions. It should be noted that BL.thresh produces singular estimates of $\Sigma_{N 100}$ (for all 1000 replicates) and hence Stein's loss $(L S)$ is not defined for BL.thresh.

As expected, the performance of these estimators is nuanced and there is no uniformly best estimator. In fact, all estimators (except the IMPLE) perform poorly under certain loss functions for certain covariance structures. For example, LW1 performs poorly for $\Sigma_{1}$ under $L S, L Q$ and $E V S$; for $\Sigma_{2}$ under $L Q$ and $E V S$; for $\Sigma_{4}$ under $L 2$; for $\Sigma_{N 100}$ under all losses except $L F$. LW2 performs poorly for $\Sigma_{1}$ under $L S, L Q, L 1$ and $L 2$; for $\Sigma_{2}$ under $L F, L 1, L 2$ 
and $E V 1$; for $\Sigma_{4}$ under $L F, L 2$ and $E V 1$. Stein's performs poorly for $\Sigma_{1}$ under $L 1$ and $L 2$; for $\Sigma_{2}$; for $\Sigma_{4}$ under $L F, E V 1, L 1$ and $L 2$; for $\Sigma_{N 100}$ under $L F$. BL.band performs poorly for $\Sigma_{1}, \Sigma_{2}, \Sigma_{4}$ and $\Sigma_{N 100}$ under $L S$ and $L Q$. BL.thresh does not show much improvement over $S$ for $\Sigma_{1}$ and $\Sigma_{2}$ under all loss functions, for $\Sigma_{4}$ under most loss functions and produces singular estimates for $\Sigma_{N 100}$. Finally the IMMLE performs poorly for $\Sigma_{1}$ under $L Q$. The IMPLE demonstrates reasonable performance for all five covariance structures under all seven loss functions.

\subsection{Positive PRIAL}

To evaluate the overall performance of an estimator (in terms of risk) compared to $S$, we can count the number of times each of the seven estimators has a positive PRIAL (rounded to two decimal places) under each of the seven loss functions, counting over the five covariance structures $\left(\Sigma_{1}, \Sigma_{2}, \Sigma_{3}, \Sigma_{4}\right.$ and $\Sigma_{N 100}$ ) and various values of $p$ and $N$, which results in 34 cases (eight cases for each $\Sigma_{i}, i=1, \ldots, 4$, and two for $\left.\Sigma_{N 100}\right)$. Table 1 reports the number of times each of the seven estimators under each of the seven loss functions has a positive PRIAL (as a percentage). According to this metric, the IMPLE, which has $100 \%$ positive PRIAL in six out of the seven loss functions is the best; followed by the IMMLE and Stein's, which have $100 \%$ positive PRIAL in three out of seven loss functions.

Table 1: Percentage(\%) positive PRIAL

\begin{tabular}{lrrrrrrr}
\hline & LW1 & LW2 & Stein & BL.band & BL.thresh & IMMLE & IMPLE \\
\hline LS & 64.71 & 79.41 & $\mathbf{1 0 0}$ & 50 & 71.88 & $\mathbf{1 0 0}$ & $\mathbf{1 0 0}$ \\
LQ & 50 & 61.76 & $\mathbf{1 0 0}$ & 41.18 & 79.41 & 67.65 & $\mathbf{1 0 0}$ \\
LF & $\mathbf{1 0 0}$ & 52.94 & 55.88 & 70.59 & 91.18 & $\mathbf{1 0 0}$ & $\mathbf{1 0 0}$ \\
L1 & 94.12 & 35.29 & 38.24 & 79.41 & 94.12 & 97.06 & $\mathbf{1 0 0}$ \\
L2 & 76.47 & 32.35 & 35.29 & 76.47 & 91.18 & 94.12 & $\mathbf{1 0 0}$ \\
EV1 & 61.76 & 38.24 & 47.06 & 70.59 & 85.29 & 76.47 & 70.59 \\
EVS & 52.94 & 94.12 & $\mathbf{1 0 0}$ & 64.71 & 47.06 & $\mathbf{1 0 0}$ & $\mathbf{1 0 0}$ \\
\hline
\end{tabular}

Entries indicate the percentage of times an estimator has positive PRIAL over various covariance structures and values of $p$ and $N$.

\section{Breast cancer Linear Discriminant Analysis (LDA) example}

In this section we demonstrate the performance of the IMMLE and the IMPLE and compare it to that of the other six estimators in a real data example. To this end, we consider a classification problem based on linear discriminant analysis, with the eight estimators plugged-in for the common covariance matrix of the classes and utilize a 30-probeset multigene predictor of pathologic complete response (pCR) to chemotherapy of 133 patients with stage I-III breast cancer that was proposed by Hess and colleagues [11]. As in [11], we split the 

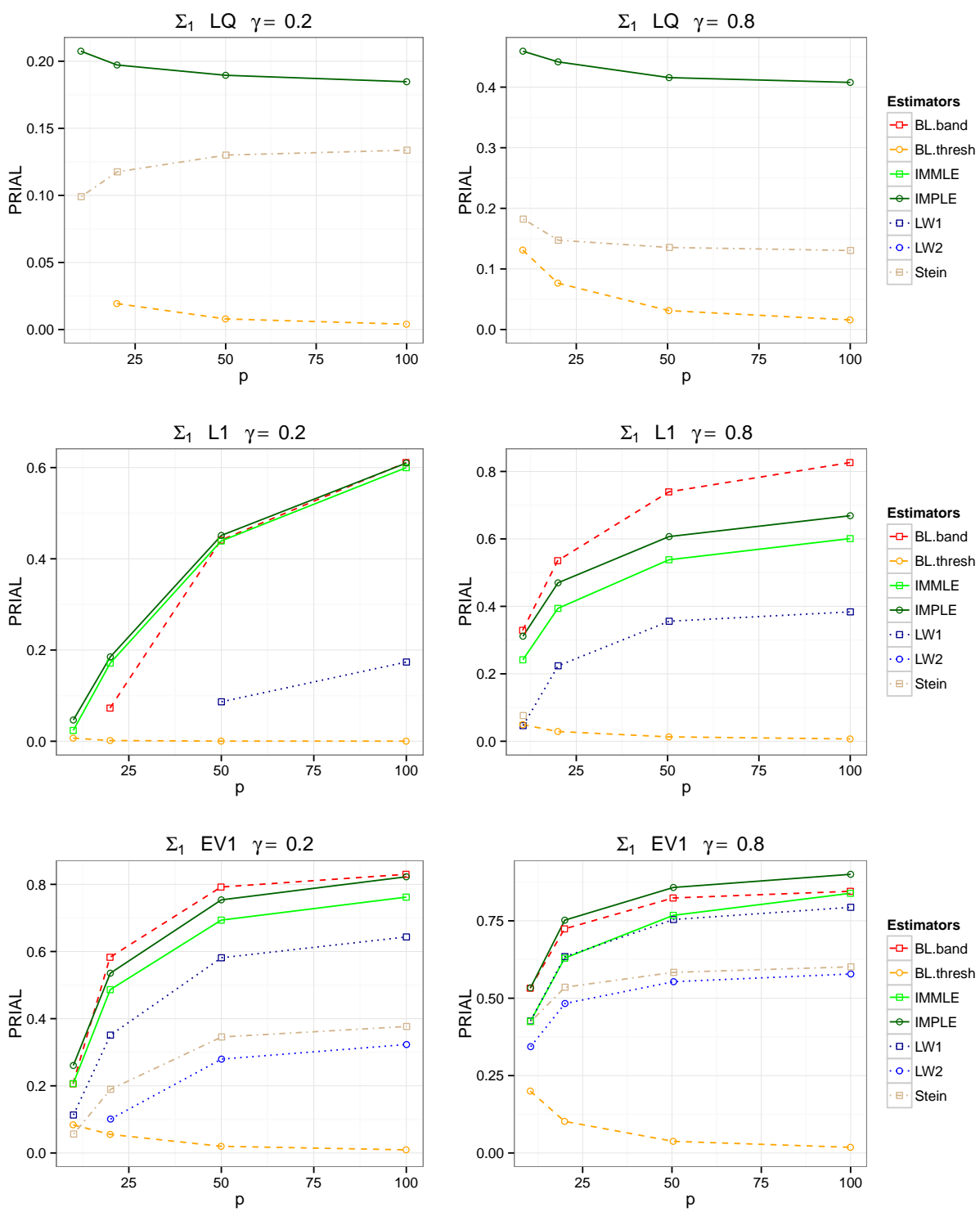

Figure 2: $\boldsymbol{\Sigma}_{\mathbf{1}}$ : PRIAL comparison of estimators. PRIAL for the estimators are plotted for $\gamma=0.2$ (left column) and $\gamma=0.8$ (right column), and three (representative) loss functions: LQ, L1, EV1 (first, second, third row). Negative PRIAL values (estimators worse than S) are not shown (see Appendix D). 

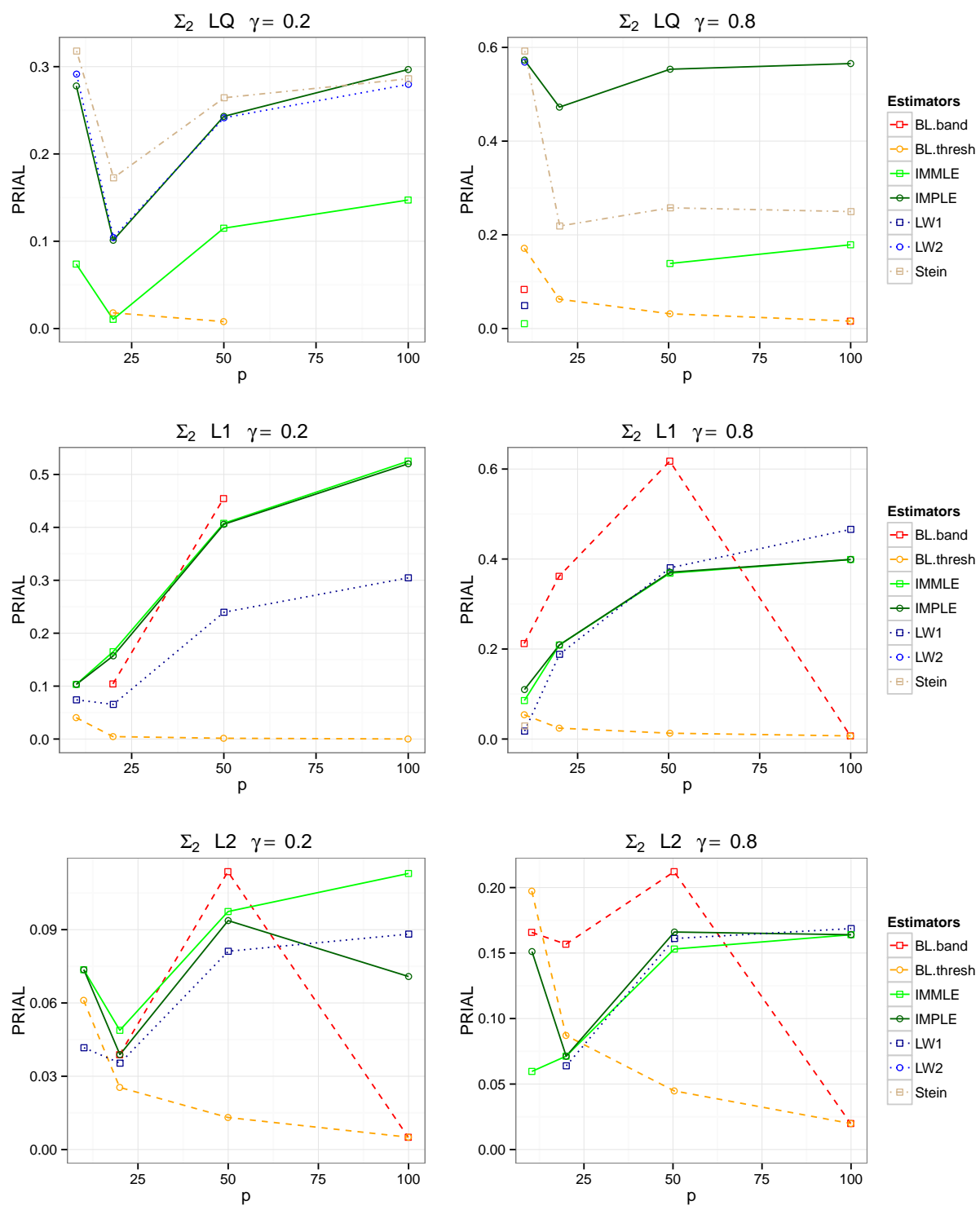

Figure 3: $\boldsymbol{\Sigma}_{\mathbf{2}}$ : PRIAL comparison of estimators. PRIAL for the estimators are plotted for $\gamma=0.2$ (left column) and $\gamma=0.8$ (right column), and three (representative) loss functions: LQ, L1, L2 (first, second, third row). Negative PRIAL values (estimators worse than S) are not shown (see Appendix D). 

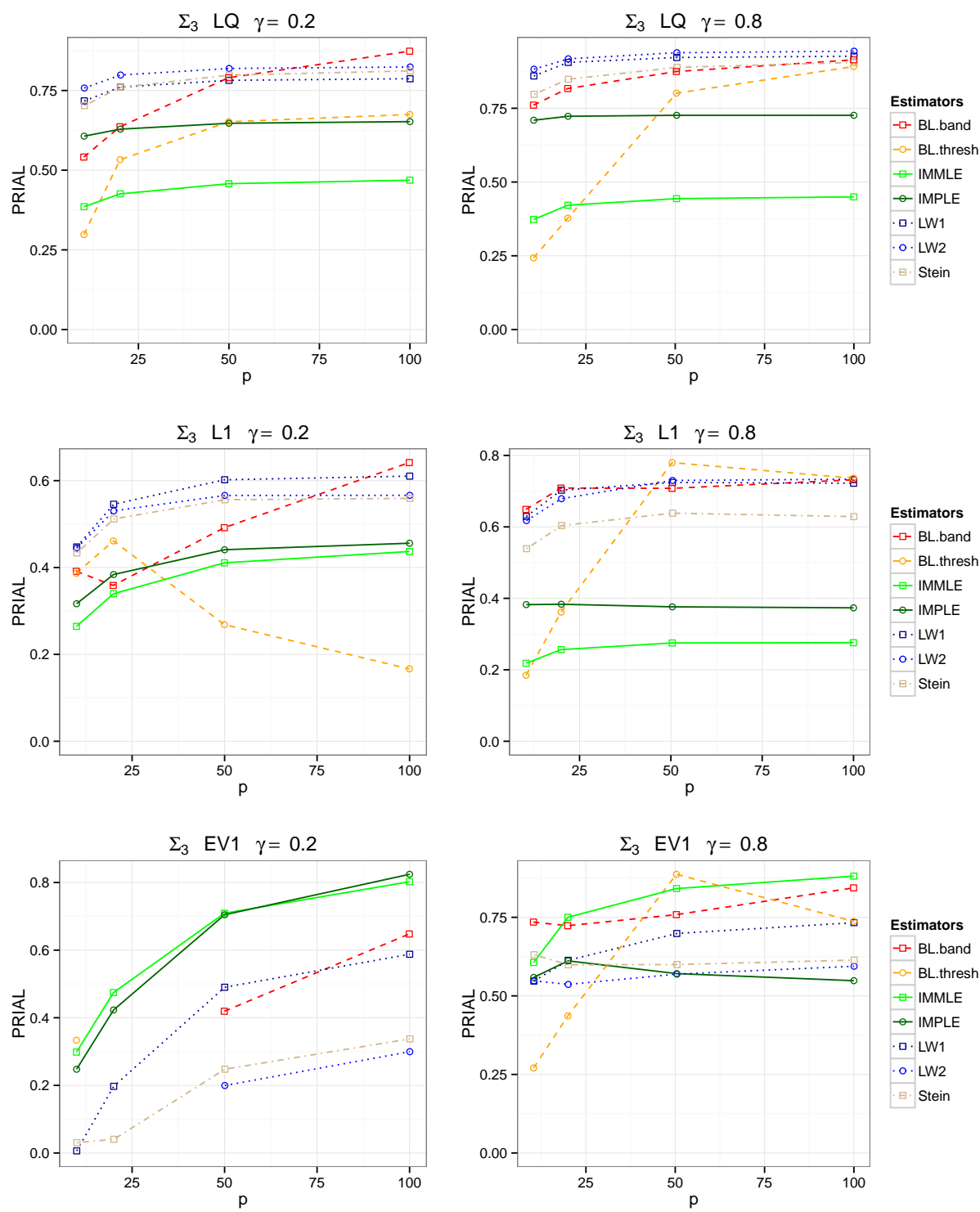

Figure 4: $\boldsymbol{\Sigma}_{\mathbf{3}}$ : PRIAL comparison of estimators. PRIAL for the estimators are plotted for $\gamma=0.2$ (left column) and $\gamma=0.8$ (right column), and three (representative) loss functions: LQ, L1, EV1 (first, second, third row). Negative PRIAL values (estimators worse than S) are not shown (see Appendix D). 

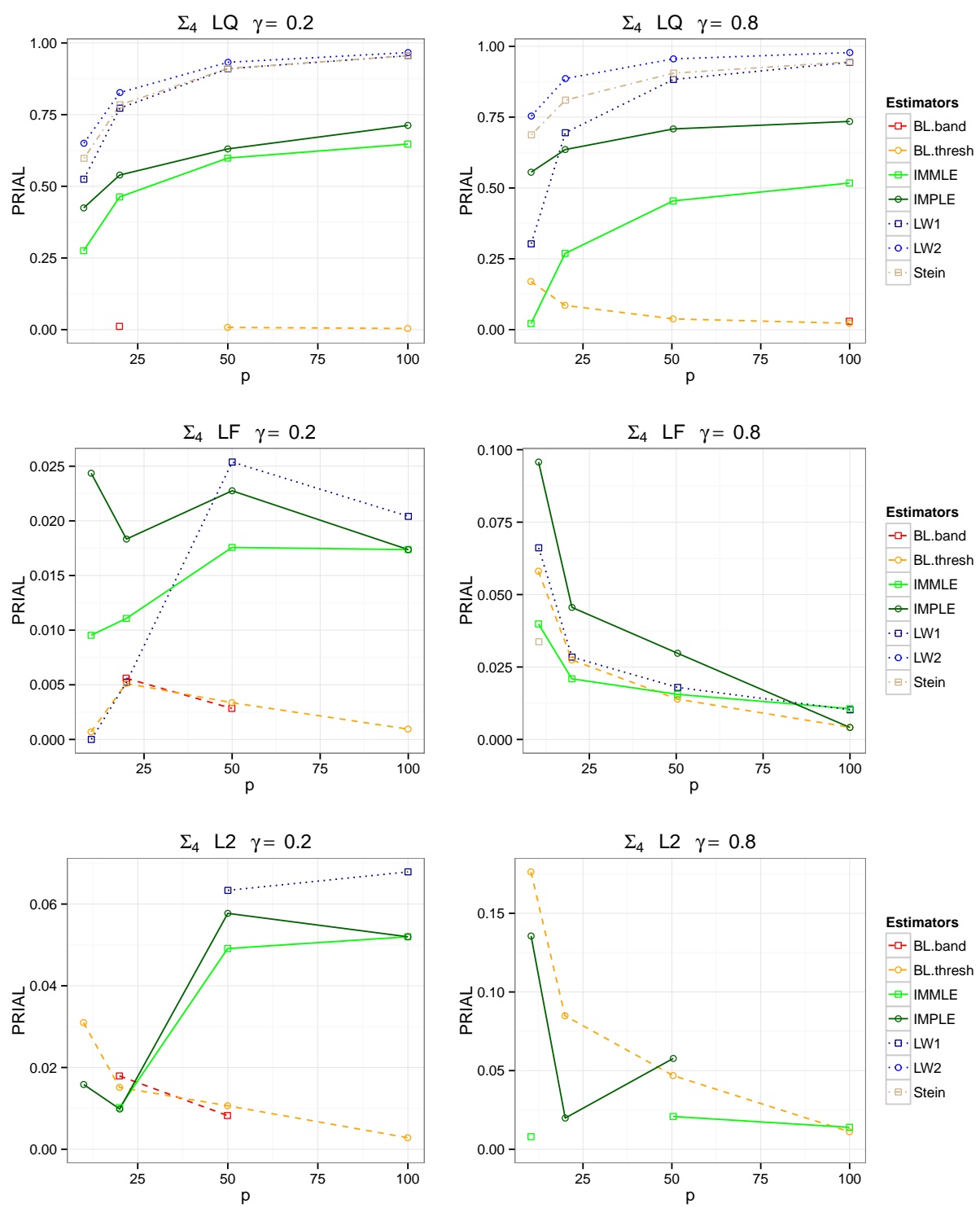

Figure 5: $\boldsymbol{\Sigma}_{\mathbf{4}}$ : PRIAL comparison of estimators. PRIAL for the estimators are plotted for $\gamma=0.2$ (left column) and $\gamma=0.8$ (right column), and three (representative) loss functions: LQ, LF, L2 (first, second, third row). Negative PRIAL values (estimators worse than S) are not shown (see Appendix D). 

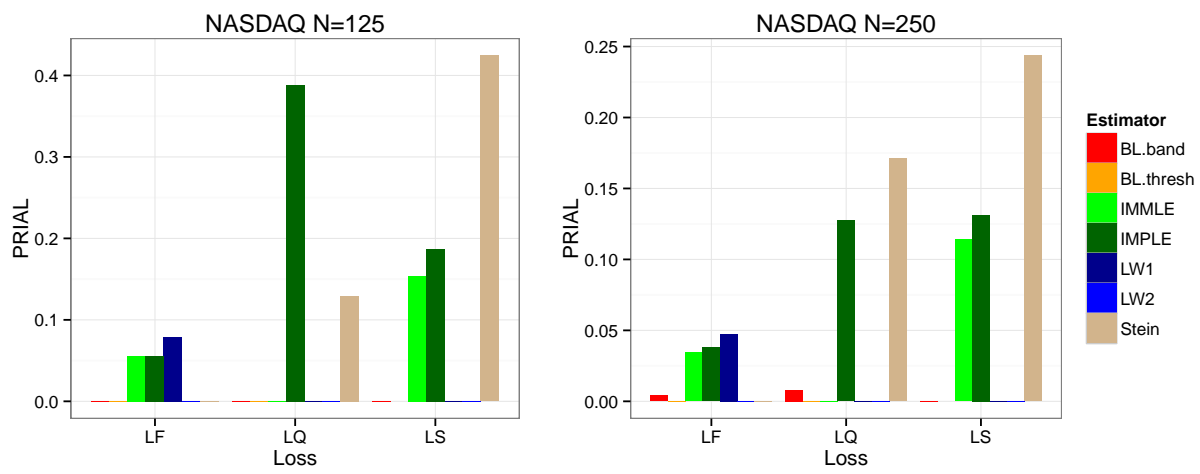

Figure 6: NASDAQ: PRIAL of seven estimators for matrix loss functions $L S, L Q$ and $L F$; negative values are omitted and hence the corresponding estimators are missing.

samples into a training set of size 82 and a test set of size 51 . We develop our classifier in the training set using the 30-probeset predictor [11]. While Hess and colleagues used Diagonal Linear Discriminant Analysis (DLDA), we use standard LDA but plug-in each of the eight estimators, studied in Section 5 , for the covariance matrix of the two pCR classes. We then evaluate and compare discriminating ability using mis-classification rate (MCR), sensitivity (Sens) and specificity (Spec), for each estimator as presented in Table 2, where $S$ represents the pooled sample covariance matrix. We should note that for the IMPLE we chose the best performing one among various $\kappa$-determining methods and that BL.thresh was singular and thus LDA could not be carried out using such estimate. The IMPLE beats all other estimators given that it has the lowest MCR and highest sensitivity and specificity. In fact, the IMPLE improves specificity by $16 \%$ when compared to the next best estimator, which is $L W 1$. Hess and colleagues [11] compared DLDA with other methods (e.g. various support vector machines and $k$-nearest neighbor algorithms) and found DLDA to produce best classification properties $(\mathrm{MCR}=0.26$, Sensitivity $=0.92$ and Specificity $=$ 0.71). The IMPLE improves specificity and MCR by $11 \%$ when compared to DLDA. 
Table 2: Mis-classification Rate, Sensitivity and Specificity of seven estimators for predicting $\mathrm{pCR}$ in the Breast Cancer example using a multigene predictor. BL.thresh is omitted because the estimate is singular.

\begin{tabular}{rccc}
\hline Estimator & MCR & Sens & Spec \\
\hline S & 0.37 & 0.85 & 0.55 \\
Stein & 0.35 & 0.92 & 0.55 \\
BL.band & 0.37 & 0.85 & 0.55 \\
LW1 & 0.33 & 0.69 & 0.66 \\
LW2 & 0.35 & 0.77 & 0.61 \\
IMMLE & 0.35 & 0.77 & 0.61 \\
IMPLE & 0.16 & 0.92 & 0.82 \\
\hline
\end{tabular}

\section{Conclusions}

In this paper, we have considered the estimation of a covariance matrix in situations in which the usual sample covariance matrix $S$ is well-known to have an overdispersed eigenspectrum, particularly when the order of the covariance matrix $(p)$ is large compared to the sample size $(N)$ but $p<N=n+1$. We have constructed two orthogonally equivariant estimators. The central idea to the proposed estimation procedure is to consider the joint distribution of the eigenvalues of $S$ by integrating out the eigenvectors with respect to the Haar measure on the orthogonal group. This marginalization takes into account all possible spatial directions in the estimation of the eigenvalues. Since the marginal likelihood of eigenvalues is not analytically tractable, its Laplace approximation is considered, which is the modified profile likelihood. The resulting maximum likelihood solutions reduce bias in eigenvalue estimation (compared to that of $S$ ) from $O\left(n^{-1}\right)$ to $O\left(n^{-2}\right)$. However, these eigenvalue MLE's are not guaranteed to be positive and ordered. To solve this problem, we have employed isotonization to ensure the ordering of the eigenvalues and have shown that the resulting estimator (the IMMLE) is positive definite. We have also proposed a second estimator (the IMPLE) which regularizes the trace of the sample covariance matrix by adding a penalty function to the modified profile likelihood. We have obtained maximum likelihood estimators from this penalized likelihood. As before, the eigenvalue estimates are not guaranteed to be positive or ordered and an isotonizing step was used. In this case too, we show that the resulting estimator (the IMPLE) is positive definite. A practical problem is to determine the intensity parameter $\kappa$ for this penalty function. To this end, we have implemented a selective-bootstrap resampling scheme. Higher order approximations for the IMMLE and the IMPLE were also obtained but not discussed in details as they do not show substantial improvement in performance compared to the lower-order approximations. We have evaluated the finite sample properties of our estimators by risk computations. We have been able to show (analytically) risk dominance of the IMMLE in a few very special cases. For a more general comparison Monte Carlo estimates of risk were computed under 
a variety of loss functions and different covariance structures (widely spaced eigenvalues, one large eigenvalue, autoregressive of order 1, spiked covariance structure and NASDAQ-100 data) which are representative of many common and real applications. We have compared the IMMLE and the IMPLE with six other estimators, the sample covariance matrix, Stein's estimator [33], two nonlinear shrinkage estimators introduced by Ledoit and Wolf [18, 19], Bickel and Levina's banded estimator [6] and Bickel and Levina's thresholded estimator [5]. The IMPLE (followed by the IMMLE) shows substantial risk reduction over all loss functions and different covariance structures. While other estimators tend to assume an underlying structure of the true covariance matrix or perform well under a specific loss function, the IMMLE and especially the IMPLE are a robust alternative when the structure is unknown and various loss functions are considered.

\section{Acknowledgments}

We would like to thank O. Ledoit and M. Wolf for providing us with the Matlab functions for their nonlinear shrinkage estimators. SB was supported by CTSC grant UL1-RR024996 and MTW by NSF grant DMS-1208488 for their efforts. 


\section{Appendix A}

In this Appendix we deduce the main properties of the IMMLE and the IMPLE, and other results which are needed for the main proofs.

Lemma 1. Let $A_{i}$ be as in (3), then

$$
\sum_{i=J}^{p} l_{i} A_{i} \geq 0
$$

with equality if and only if $J=1$.

Proof. By definition of $A_{i}$

$$
\sum_{i=J}^{p} l_{i} A_{i}=\sum_{i=J}^{p} l_{i} \sum_{j \neq i} \frac{l_{j}}{l_{j}-l_{i}} .
$$

When $J=1$ the ranges of values of $i$ and $j$ coincide, but the summands are antisymmetric under the exchange of the indices $i, j$. If $J>1$,

$$
\begin{aligned}
\sum_{i=J}^{p} l_{i} \sum_{j \neq i} \frac{l_{j}}{l_{j}-l_{i}} & =\sum_{i=J}^{p} l_{i}\left(\sum_{j=1}^{J-1} \frac{l_{j}}{l_{j}-l_{i}}+\sum_{j=J, \neq i}^{p} \frac{l_{j}}{l_{j}-l_{i}}\right) \\
& =\sum_{i=J}^{p} l_{i} \sum_{j=1}^{J-1} \frac{l_{j}}{l_{j}-l_{i}}>0
\end{aligned}
$$

because the second term in the right hand side of the first line is antisymmetric under the exchange of the indices $i, j$, and $l_{j}>l_{i}$ for $j<i$.

Lemma 2. For all $J=1, \ldots, p$

$$
\sum_{i=J}^{p} \tilde{\lambda}_{i}>0
$$

Proof. By Lemma 1 it follows that

$$
\sum_{i=J}^{p} \tilde{\lambda}_{i}=\sum_{i=J}^{p} l_{i}\left(1+A_{i} / n\right)=\sum_{i=J}^{p} l_{i}+\sum_{i=J}^{p} l_{i} A_{i} / n>0 .
$$

Let us fix notation for the following lemma and proposition. Let $(\pi(1), \ldots, \pi(K))$ be the isotonic blocks, with $\pi(j)=\left\{i: m_{j-1}+1 \leq i \leq m_{j}\right\}$, and thus, $\hat{\lambda}_{i}=\hat{\lambda}_{j}$ for all $i, j \in \pi(s)$ with $s=\{1, \ldots, K\}, m_{0}=0$, and $m_{K}=p$.

Lemma 3. The following inequality holds true for all $s=1, \ldots, K-1$

$$
\sum_{i=1}^{m_{s}} \hat{\lambda}_{i}<\sum_{i=1}^{m_{s}} l_{i}
$$


Proof. By definition of isotonic regression, we have

$$
\sum_{i \in \pi(m)} \tilde{\lambda}_{i}=\sum_{i \in \pi(m)} \hat{\lambda}_{i}
$$

which can be larger than, or smaller than, or equal to $\sum_{i \in \pi(m)} l_{i}$. Then

$$
\begin{aligned}
\sum_{i=1}^{m_{s}} l_{i}-\sum_{i=1}^{m_{s}} \hat{\lambda}_{i} & =\sum_{i=1}^{m_{s}} \tilde{\lambda}_{i}-\sum_{i=1}^{m_{s}} \hat{\lambda}_{i}+\frac{1}{n} \sum_{i=m_{s}+1}^{p} l_{i} A_{i} \\
& =\frac{1}{n} \sum_{i=m_{s}+1}^{p} l_{i} A_{i}>0
\end{aligned}
$$

because of (11) applied to the union of consecutive blocks $\pi$ 's and by Lemma 1.

Proof of Proposition 1. The decreasing ordering of the estimates is guaranteed by the solution (5) of the isotonic regression. As for the positivity of the estimates, it is sufficient to show that $\hat{\lambda}_{p}>0$. Applying (5), we have $\hat{\lambda}_{p}=\min _{s \leq p}\left\{\sum_{r=s}^{p} \tilde{\lambda}_{r} /(p-s+1)\right\}$, which is positive because of Lemma 2, which proves part a.

Since $\sum_{i=1}^{p} \tilde{\lambda}_{i}=\sum_{i=1}^{p} l_{i}+\frac{1}{n} \sum_{i=1}^{p} l_{i} A_{i}=\sum_{i=1}^{p} l_{i}$ by Lemma 1 , and the isotonic regressions preserve the sums, $\sum_{i=1}^{p} \hat{\lambda}_{i}=\sum_{i=1}^{p} \tilde{\lambda}_{i}$, we have $\sum_{i=1}^{p} \hat{\lambda}_{i}=$ $\sum_{i=1}^{p} l_{i}$, which proves part b.

Suppose there existed a $J, m_{s}<J<m_{s+1}$, such that $\sum_{i=1}^{J} \hat{\lambda}_{i} \geq \sum_{i=1}^{J} l_{i}$, then $\sum_{i=m_{s}+1}^{J} \hat{\lambda}_{i}>\sum_{i=m_{s}+1}^{J} l_{i}$ because of Lemma 3. Since $l_{i}>l_{j}$ for $i<j$, $\hat{\lambda}_{\pi(s+1)}>l_{J}$ where $\hat{\lambda}_{\pi(s+1)}$ is the common value of the estimator for indices inside the block $\pi(s+1)$ where $J$ falls, and thus $\sum_{i=J+1}^{m_{s+1}} \hat{\lambda}_{i}>\sum_{i=J+1}^{m_{s+1}} l_{i}$. $\sum_{i=1}^{J} \hat{\lambda}_{i} \geq \sum_{i=1}^{J} l_{i}$ would then imply $\sum_{i=1}^{m_{s+1}} \hat{\lambda}_{i}>\sum_{i=1}^{m_{s+1}} l_{i}$, which contradicts Lemma 3 , and thus part $\mathrm{c}$ is proven

Lemma 4. For $\kappa<n$ it follows that

$$
\sum_{i=J}^{p} \tilde{\lambda}_{i}^{\kappa}>0
$$

Proof. When $\kappa \leq 0$, the inequality is an immediate consequence of Lemma 1. Assume now $\kappa>0$. After multiplying by $n \operatorname{tr}\left(S^{2}\right)$, the above inequality is equivalent to

$$
n \operatorname{tr}\left(S^{2}\right) \sum_{i=J}^{p} l_{i}+\operatorname{tr}\left(S^{2}\right) \sum_{i=J}^{p} l_{i} A_{i}-\kappa \operatorname{tr}(S) \sum_{i=J}^{p} l_{i}^{2}>0 .
$$


Since

$$
\begin{aligned}
n \operatorname{tr}\left(S^{2}\right) \sum_{i=J}^{p} l_{i}-\kappa \operatorname{tr}(S) \sum_{i=J}^{p} l_{i}^{2} & = \\
(n-\kappa) \sum_{k=J}^{p} l_{k}^{2} \sum_{i=J}^{p} l_{i}+n \sum_{k=1}^{J-1} l_{k}^{2} \sum_{i=J}^{p} l_{i}-\kappa \sum_{k=1}^{J-1} l_{k} \sum_{i=J}^{p} l_{i}^{2} & > \\
n\left(\sum_{k=1}^{J-1} l_{k}^{2} \sum_{i=J}^{p} l_{i}-\sum_{k=1}^{J-1} l_{k} \sum_{i=J}^{p} l_{i}^{2}\right) & = \\
n \sum_{k=1}^{J-1} l_{k} \sum_{i=J}^{p}\left(l_{k} l_{i}-l_{i}^{2}\right) & >0
\end{aligned}
$$

because $n>\kappa$ and $k<J \leq i$, and Lemma 1, the result follows.

Proof of Proposition 3. Part a follows from Lemma 4 and by using the same argument as in the proof of Proposition 1.

It is plain to see that $\tilde{\lambda}_{i}^{\kappa}<\tilde{\lambda}_{i}^{0}, i=1, \ldots, p$, when $\kappa>0$. Since the isotonic regression preserves the order [29], the result $\mathrm{b}$ follows.

\section{Appendix B}

In this Appendix we compare $S$ and the IMMLE $\hat{\Sigma}^{0}$ using the theoretical expressions of risk differences in special cases. We assume $\Sigma$ to be diagonal, without loss of generality.

Proof of Proposition 4. Using the fact that $\hat{\lambda}^{0}$ is majorized by $l$, we have

$$
L 1\left(\hat{\Sigma}^{0}, \Sigma\right)-L 1(S, \Sigma)=\left(\sum_{i=1}^{p}\left|\hat{\lambda}_{i}^{0}-c\right|-\sum_{i=1}^{p}\left|l_{i}-c\right|\right) / p \leq 0
$$

and

$$
L 2\left(\hat{\Sigma}^{0}, \Sigma\right)-L 2(S, \Sigma)=\left(\sum_{i=1}^{p}\left(\hat{\lambda}_{i}^{0}-c\right)^{2}-\sum_{i=1}^{p}\left(l_{i}-c\right)^{2}\right) / p<0
$$

(strict inequality since since $\hat{\lambda}^{0}$ is not a permutation of $l$ ). Similarly, for the quadratic loss,

$$
L Q\left(\hat{\Sigma}^{0}, \Sigma\right)-L Q(S, \Sigma)=\left(\sum_{i=1}^{p}\left(\hat{\lambda}_{i}^{0} / c-1\right)^{2}-\sum_{i=1}^{p}\left(l_{i} / c-1\right)^{2}\right) / p<0 .
$$

Thus taking the expectations, the proof follows. Under Stein's loss (see [31])

$$
R\left(\hat{\Sigma}^{0}, \Sigma\right)-R(S, \Sigma)=E_{\Sigma}\left(\sum_{i=1}^{p}\left(\hat{\lambda}_{i}^{0}-l_{i}\right) E_{\Sigma}\left(a_{i} \mid L\right)\right)-E_{\Sigma}\left(\log \left(\operatorname{det} \hat{\Sigma}^{0}\right)-\log (\operatorname{det} S)\right)
$$


where $a_{i}=\left(H^{T} \Sigma^{-1} H\right)_{i i}, E_{\Sigma}\left(a_{i} \mid L\right) \propto \int_{O(p)} a_{i} \exp \left(-(n / 2) \operatorname{tr}\left(\Sigma^{-1} H L H^{T}\right)\right)(d O)$. Because of the majorization property, the second term is negative and the first term vanishes when $\Sigma=c I$ (since then $a_{i}$ is the same for all $i$ ).

We now focus on Stein's loss $L S$ and compare the IMMLE with $S$. We are able to obtain some inequalities under which the IMMLE dominates $S$. The two specific cases considered are those in which the IMMLE eigenspectrum has maximum degeneracy (i.e. one distinct eigenvalue with multiplicity $p$ ) or is not degenerate (i.e. all its eigenvalues are distinct). The results below are based on the estimated risk due to Haff and Stein [34].

One distinct eigenvalue

Let us consider the case when the IMMLE eigenspectrum has maximum degeneracy. Thus $\hat{\lambda}_{i}=\frac{\sum_{i=1}^{p} l_{i}}{p}$ for all $i$. Let us indicate with $\hat{\Sigma}^{K=1}$ the corresponding IMMLE. Three conditions under which $\hat{\Sigma}^{K=1}$ is better than $S$ under Stein's loss are given by the following

Result 1. Under the following conditions,

a) $n>p+1$ and $\operatorname{tr}(S) \operatorname{tr}\left(S^{-1}\right)<p \frac{n p-2}{n-p-1}$;

b) $n=p+1 \geq 3$ and for all $S$;

c) $n=p \geq 2$ and for all $S$;

$\hat{\Sigma}^{K=1}$ dominates $S$ under Stein's loss.

Proof. By definition of Stein's loss we have

$$
R\left(\hat{\Sigma}^{K=1}, \Sigma\right)=2+\frac{n-p-1}{p} \sum_{i=1}^{p} l_{i} \cdot \sum_{i=1}^{p} \frac{1}{l_{i}}-\sum_{i} \log \hat{\lambda}_{i},
$$

and thus

$$
R\left(\hat{\Sigma}^{K=1}, \Sigma\right)-R(S, \Sigma) \leq 2+\frac{n-p-1}{p} \sum_{i=1}^{p} l_{i} \cdot \sum_{i=1}^{p} \frac{1}{l_{i}}-n p .
$$

By Jensen's inequality

$$
\sum_{i=1}^{p} \log \hat{\lambda}_{i}=p \log \frac{\sum_{i=1}^{p} l_{i}}{p} \geq \sum_{i=1}^{p} \log l_{i}
$$

hence the result follows.

Non-degenerate eigenspectrum

Let us now consider the other extreme case, when all $p$ eigenvalues of the IMMLE, $\hat{\Sigma}^{K=p}$, are distinct: $\hat{\lambda}_{i}=\tilde{\lambda}_{i}$, for all $i$. For this case to be possible, the solutions to our ML equations must be positive and ordered, and thus

$$
l_{i}-l_{j}>\frac{A_{j} l_{j}-l_{i} A_{i}}{n} \quad j>i
$$

and $1+A_{i} / n>0$, for all $i$. 
Result 2. Under the following condition

$\frac{1}{n} \sum_{\substack{i, j=1 \\ i \neq j}}^{p} \sum_{\substack{m=1 \\ m \neq i, j}}^{p} \frac{l_{m}^{2}}{\left(l_{i}-l_{m}\right)\left(l_{j}-l_{m}\right)}-\sum_{i=1}^{p} \log \left(1+\sum_{j \neq i} \frac{l_{j}}{n\left(l_{j}-l_{i}\right)}\right)<-\frac{n-p+1}{n}\left(\begin{array}{l}p \\ 2\end{array}\right)$

$\hat{\Sigma}^{K=p}$ dominates $S$ with respect to Stein's loss.

Proof. We have

$$
\begin{aligned}
\sum_{i} \frac{\tilde{\lambda}_{i}}{l_{i}} & =p+\frac{p(p-1)}{2 n} \\
2 \sum_{i} \frac{\partial \tilde{\lambda}_{i}}{\partial l_{i}} & =2 p+\frac{2}{n}\left(\begin{array}{l}
p \\
2
\end{array}\right)+\frac{2}{n} \sum_{i, j=1, i \neq j}^{p} \frac{l_{i} l_{j}}{\left(l_{i}-l_{j}\right)^{2}}
\end{aligned}
$$

and thus the difference of the risk estimates is

$$
\begin{aligned}
R\left(\hat{\Sigma}^{K=p}, \Sigma\right)-R(S, \Sigma)= & \frac{2}{n} \sum_{i=1}^{p-1} \sum_{j=i+1}^{p} \frac{l_{i} A_{i}-l_{j} A_{j}}{l_{i}-l_{j}}-\sum_{i=1}^{p} \log \left(1+\frac{A_{i}}{n}\right) \\
& +\frac{n-p+1}{n}\left(\begin{array}{l}
p \\
2
\end{array}\right)+\frac{2}{n} \sum_{\substack{i, j=1 \\
i \neq j}}^{p} \frac{l_{i} l_{j}}{\left(l_{i}-l_{j}\right)^{2}} .
\end{aligned}
$$

It is easy to see that this difference can be re-written as

$$
\begin{aligned}
R\left(\hat{\Sigma}^{K=p}, \Sigma\right)-R(S, \Sigma)= & \frac{1}{n} \sum_{\substack{i, j=1 \\
i \neq j}}^{p} \sum_{\substack{m=1 \\
m \neq i, j}}^{p} \frac{l_{m}^{2}}{\left(l_{i}-l_{m}\right)\left(l_{j}-l_{m}\right)}-\sum_{i=1}^{p} \log \left(1+\frac{A_{i}}{n}\right) \\
& +\frac{n-p+1}{n}\left(\begin{array}{l}
p \\
2
\end{array}\right)
\end{aligned}
$$

from which the result follows.

\section{Appendix C}

In this Appendix we derive the solutions to the maximum likelihood equations for the penalized modified profile likelihood (7). These equations are given in eq. (8) and are reported here for convenience:

$$
\begin{aligned}
l_{i} & =\lambda_{i}+\frac{\lambda_{i}}{n} \sum_{j \in I_{m}^{\prime}} \frac{\lambda_{j}}{\lambda_{i}-\lambda_{j}}-\lambda_{i}^{2} \frac{2}{n} \mu, \\
\sum_{i} \lambda_{i} & =\sum_{i} l_{i}\left(1-\frac{\kappa}{n}\right) .
\end{aligned}
$$


Proof of Proposition 2. By summing (12) for all $i$ and using (13), we find $-2 \mu=\kappa \operatorname{tr}(S) / \operatorname{tr}\left(\Sigma^{2}\right)$. Thus we need to solve:

$$
l_{i}=\lambda_{i}+\frac{\lambda_{i}}{n} \sum_{j \in I_{m}^{\prime}} \frac{\lambda_{j}}{\lambda_{i}-\lambda_{j}}+\lambda_{i}^{2} \frac{\kappa}{n} \frac{\operatorname{tr}(S)}{\operatorname{tr}\left(\Sigma^{2}\right)}, \quad i=1, \ldots, n .
$$

We consider the Ansatz $\tilde{\lambda}_{i}=a_{i} l_{i}+b_{i} f_{i}(l) / n+O\left(n^{-2}\right)$ which guesses the form of the solutions to the ML equations, with $a_{i}, b_{i} \in \mathbb{R}$ and $f_{i}$ functions of $l$. Such a form is based on Anderson's solution. Plugging $\tilde{\lambda}_{i}$ in (14), Taylor-expanding in $n$ up to $O\left(n^{-2}\right)$, and equating the coefficients of the polynomials at either side of (14), we obtain the unknown constants. In more detail, let $T_{1}, T_{2}$ and $T_{3}$ be the terms on the right hand side of (14). We have

$$
\begin{aligned}
T_{1} & =a l_{i}+\frac{1}{n} b_{i} f_{i}+O\left(n^{-2}\right), \\
T_{2} & =\frac{a}{n} l_{i} \sum_{j \in I_{m}^{\prime}} \frac{l_{j}}{l_{i}-l_{j}}+O\left(n^{-2}\right), \\
T_{3} & =\frac{a \kappa}{n} \frac{\operatorname{tr}(S)}{\operatorname{tr}\left(S^{2}\right)} l_{i}^{2}+O\left(n^{-2}\right),
\end{aligned}
$$

so that, when we equate the terms of order 0 and 1 in $n$, we find $a=1$ and $b_{i} f_{i}(l)=l_{i} A_{i}-\kappa \frac{\operatorname{tr}(S)}{\operatorname{tr}\left(S^{2}\right)} l_{i}^{2}$.

\section{Appendix D}

In this Appendix we present the detailed PRIAL tables for each of the five covariance structures used to simulate data (see Section 5.3). 
Table 3: PRIAL $\left(\boldsymbol{\Sigma}_{\mathbf{1}}\right)$ : Proportion Reduction in Integrated Average Loss of seven estimators compared to $S$ for various loss functions, and values for $p$ and $\gamma$.

\begin{tabular}{|c|c|c|c|c|c|c|c|c|c|}
\hline \multirow[t]{3}{*}{ Loss } & \multicolumn{2}{|l|}{$\gamma(p / N)$} & \multicolumn{3}{|c|}{0.2} & \multicolumn{4}{|c|}{0.8} \\
\hline & $\mathrm{p}$ & 10 & 20 & 50 & 100 & 10 & 20 & 50 & 100 \\
\hline & $\mathrm{n}$ & 50 & 100 & 250 & 500 & 13 & 25 & 63 & 125 \\
\hline \multirow[t]{7}{*}{ LS } & LW1 & -0.10 & -0.15 & -0.72 & -1.42 & -0.59 & -1.38 & -2.43 & -2.57 \\
\hline & LW2 & 0.08 & 0.08 & -0.32 & -0.87 & 0.01 & -0.04 & -0.15 & -0.07 \\
\hline & Stein & 0.14 & 0.15 & 0.16 & 0.16 & 0.44 & 0.42 & 0.40 & 0.40 \\
\hline & BL.band & -8.93 & -3.46 & -15.97 & -17.18 & -0.67 & 0.22 & -1.98 & -2.22 \\
\hline & BL.thresh & -0.47 & 0.00 & 0.00 & 0.00 & -0.02 & 0.00 & 0.00 & 0.00 \\
\hline & IMMLE & 0.08 & 0.09 & 0.10 & 0.10 & 0.18 & 0.19 & 0.19 & 0.19 \\
\hline & IMPLE & 0.15 & 0.16 & 0.16 & 0.16 & 0.27 & 0.28 & 0.29 & 0.29 \\
\hline \multirow[t]{7}{*}{ LQ } & LW1 & -0.69 & -0.86 & -3.98 & -16.48 & -14.61 & -38.89 & -119.09 & -197.37 \\
\hline & LW2 & -0.04 & -0.08 & -1.96 & -9.90 & -5.85 & -8.14 & -12.25 & -15.42 \\
\hline & Stein & 0.10 & 0.12 & 0.13 & 0.13 & 0.18 & 0.15 & 0.14 & 0.13 \\
\hline & BL.band & -80.26 & -28.42 & -660.42 & -1078.33 & -18.93 & -6.02 & -160.68 & -266.95 \\
\hline & BL.thresh & -0.61 & 0.02 & 0.01 & 0.00 & 0.13 & 0.08 & 0.03 & 0.02 \\
\hline & IMMLE & -0.06 & -0.05 & -0.05 & -0.05 & -0.09 & -0.10 & -0.12 & -0.13 \\
\hline & IMPLE & 0.21 & 0.20 & 0.19 & 0.18 & 0.46 & 0.44 & 0.42 & 0.41 \\
\hline \multirow[t]{7}{*}{$\mathrm{LF}$} & LW1 & 0.11 & 0.15 & 0.18 & 0.19 & 0.26 & 0.33 & 0.36 & 0.37 \\
\hline & LW2 & 0.10 & 0.10 & 0.11 & 0.11 & 0.26 & 0.26 & 0.24 & 0.23 \\
\hline & Stein & 0.12 & 0.12 & 0.11 & 0.11 & 0.29 & 0.27 & 0.25 & 0.24 \\
\hline & BL.band & -0.02 & 0.24 & 0.40 & 0.53 & 0.34 & 0.49 & 0.62 & 0.71 \\
\hline & BL.thresh & 0.01 & 0.01 & 0.00 & 0.00 & 0.07 & 0.04 & 0.02 & 0.01 \\
\hline & IMMLE & 0.09 & 0.11 & 0.13 & 0.14 & 0.17 & 0.21 & 0.23 & 0.25 \\
\hline & IMPLE & 0.13 & 0.14 & 0.15 & 0.15 & 0.29 & 0.29 & 0.29 & 0.29 \\
\hline \multirow[t]{7}{*}{ L1 } & LW1 & -0.21 & -0.13 & 0.09 & 0.17 & 0.05 & 0.22 & 0.36 & 0.38 \\
\hline & LW2 & -0.26 & -0.38 & -0.44 & -0.46 & -0.03 & -0.08 & -0.15 & -0.18 \\
\hline & Stein & -0.19 & -0.33 & -0.41 & -0.44 & 0.08 & -0.01 & -0.10 & -0.13 \\
\hline & BL.band & -0.25 & 0.07 & 0.44 & 0.61 & 0.33 & 0.54 & 0.74 & 0.83 \\
\hline & BL.thresh & 0.01 & 0.00 & 0.00 & 0.00 & 0.05 & 0.03 & 0.01 & 0.01 \\
\hline & IMMLE & 0.02 & 0.17 & 0.44 & 0.60 & 0.24 & 0.39 & 0.54 & 0.60 \\
\hline & IMPLE & 0.05 & 0.19 & 0.45 & 0.61 & 0.31 & 0.47 & 0.61 & 0.67 \\
\hline \multirow[t]{7}{*}{$\mathrm{L} 2$} & LW1 & -0.15 & 0.02 & 0.36 & 0.49 & 0.36 & 0.59 & 0.73 & 0.76 \\
\hline & LW2 & -0.34 & -0.64 & -0.83 & -0.93 & 0.26 & 0.16 & 0.04 & -0.01 \\
\hline & Stein & -0.20 & -0.50 & -0.76 & -0.89 & 0.39 & 0.26 & 0.11 & 0.04 \\
\hline & BL.band & -0.06 & 0.44 & 0.78 & 0.90 & 0.61 & 0.80 & 0.93 & 0.97 \\
\hline & BL.thresh & 0.08 & 0.06 & 0.03 & 0.02 & 0.22 & 0.14 & 0.06 & 0.03 \\
\hline & IMMLE & 0.12 & 0.36 & 0.70 & 0.84 & 0.42 & 0.65 & 0.82 & 0.88 \\
\hline & IMPLE & 0.21 & 0.42 & 0.73 & 0.86 & 0.64 & 0.79 & 0.90 & 0.93 \\
\hline \multirow[t]{7}{*}{ EV1 } & LW1 & 0.11 & 0.35 & 0.58 & 0.64 & 0.43 & 0.63 & 0.75 & 0.79 \\
\hline & LW2 & -0.03 & 0.10 & 0.28 & 0.32 & 0.34 & 0.48 & 0.55 & 0.58 \\
\hline & Stein & 0.06 & 0.19 & 0.35 & 0.38 & 0.42 & 0.54 & 0.58 & 0.60 \\
\hline & BL.band & 0.21 & 0.58 & 0.79 & 0.83 & 0.53 & 0.72 & 0.82 & 0.85 \\
\hline & BL.thresh & 0.08 & 0.06 & 0.02 & 0.01 & 0.20 & 0.10 & 0.04 & 0.02 \\
\hline & IMMLE & 0.21 & 0.49 & 0.69 & 0.76 & 0.43 & 0.63 & 0.77 & 0.84 \\
\hline & IMPLE & 0.26 & 0.54 & 0.75 & 0.82 & 0.53 & 0.75 & 0.86 & 0.90 \\
\hline \multirow[t]{7}{*}{ EVS } & LW1 & -0.58 & -0.48 & -0.42 & -0.36 & -1.32 & -1.07 & -1.34 & -1.29 \\
\hline & LW2 & -0.03 & 0.16 & 0.42 & 0.61 & -0.20 & 0.33 & 0.50 & 0.55 \\
\hline & Stein & 0.09 & 0.25 & 0.52 & 0.66 & 0.36 & 0.50 & 0.63 & 0.67 \\
\hline & BL.band & -2.88 & -1.75 & -0.92 & -0.87 & -0.08 & 0.35 & 0.53 & 0.53 \\
\hline & BL.thresh & -0.05 & -0.04 & -0.02 & -0.01 & -0.02 & -0.02 & -0.01 & 0.00 \\
\hline & IMMLE & 0.19 & 0.36 & 0.59 & 0.70 & 0.24 & 0.27 & 0.28 & 0.28 \\
\hline & IMPLE & 0.20 & 0.36 & 0.59 & 0.70 & 0.24 & 0.27 & 0.28 & 0.28 \\
\hline
\end{tabular}

Boldface entries correspond to the highest PRIAL for a combination of $\gamma$ and $p$ under a certain loss function. 
Table 4: PRIAL $\left(\boldsymbol{\Sigma}_{\mathbf{2}}\right)$ : Proportion Reduction in Integrated Average Loss of seven estimators compared to $S$ for various loss functions, and values for $p$ and $\gamma$.

\begin{tabular}{|c|c|c|c|c|c|c|c|c|c|}
\hline \multirow[t]{3}{*}{ Loss } & \multicolumn{2}{|l|}{$\gamma(p / N)$} & \multicolumn{3}{|c|}{0.2} & \multicolumn{4}{|c|}{0.8} \\
\hline & $\mathrm{p}$ & 10 & 20 & 50 & 100 & 10 & 20 & 50 & 100 \\
\hline & $\mathrm{n}$ & 50 & 100 & 250 & 500 & 13 & 25 & 63 & 125 \\
\hline \multirow[t]{7}{*}{$\mathrm{LS}$} & LW1 & 0.25 & 0.03 & 0.19 & 0.23 & 0.62 & -0.39 & -0.14 & 0.14 \\
\hline & LW2 & 0.32 & 0.19 & 0.31 & 0.34 & 0.67 & 0.25 & 0.35 & 0.52 \\
\hline & Stein & 0.33 & 0.21 & 0.31 & 0.34 & 0.61 & 0.48 & 0.56 & 0.59 \\
\hline & BL.band & -0.50 & -3.31 & -3.26 & -0.01 & 0.61 & -0.42 & 0.21 & 0.00 \\
\hline & BL.thresh & -0.22 & 0.00 & 0.00 & -0.03 & 0.05 & 0.03 & -0.01 & 0.00 \\
\hline & IMMLE & 0.18 & 0.14 & 0.22 & 0.24 & 0.21 & 0.21 & 0.28 & 0.29 \\
\hline & IMPLE & 0.23 & 0.16 & 0.23 & 0.25 & 0.29 & 0.24 & 0.30 & 0.34 \\
\hline \multirow[t]{7}{*}{ LQ } & LW1 & -0.01 & -0.46 & -0.19 & -0.12 & 0.05 & -12.71 & -11.04 & -5.74 \\
\hline & LW2 & 0.29 & 0.10 & 0.24 & 0.28 & 0.57 & -3.07 & -2.85 & -0.64 \\
\hline & Stein & 0.32 & 0.17 & 0.26 & 0.29 & 0.59 & 0.22 & 0.26 & 0.25 \\
\hline & BL.band & -0.74 & -23.15 & -63.12 & 0.00 & 0.08 & -17.62 & -15.45 & 0.02 \\
\hline & BL.thresh & -0.09 & 0.02 & 0.01 & -0.01 & 0.17 & 0.06 & 0.03 & 0.02 \\
\hline & IMMLE & 0.07 & 0.01 & 0.11 & 0.15 & 0.01 & -0.04 & 0.14 & 0.18 \\
\hline & IMPLE & 0.28 & 0.10 & 0.24 & 0.30 & 0.57 & 0.47 & 0.55 & 0.57 \\
\hline \multirow[t]{7}{*}{$\mathrm{LF}$} & LW1 & 0.06 & 0.04 & 0.06 & 0.07 & 0.11 & 0.06 & 0.10 & 0.09 \\
\hline & LW2 & 0.02 & -0.17 & -0.42 & -0.87 & 0.09 & -0.27 & -0.70 & -1.34 \\
\hline & Stein & 0.03 & -0.15 & -0.41 & -0.86 & 0.13 & -0.23 & -0.68 & -1.31 \\
\hline & BL.band & -0.15 & -0.03 & 0.14 & 0.00 & 0.03 & 0.14 & 0.35 & 0.01 \\
\hline & BL.thresh & -0.01 & 0.01 & 0.00 & 0.00 & 0.07 & 0.03 & 0.01 & 0.01 \\
\hline & IMMLE & 0.04 & 0.03 & 0.04 & 0.05 & 0.05 & 0.04 & 0.06 & 0.06 \\
\hline & IMPLE & 0.04 & 0.03 & 0.05 & 0.04 & 0.11 & 0.04 & 0.07 & 0.06 \\
\hline \multirow{7}{*}{ L1 } & LW1 & 0.07 & 0.07 & 0.24 & 0.30 & 0.02 & 0.19 & 0.38 & 0.47 \\
\hline & LW2 & -0.06 & -0.28 & -0.28 & -0.35 & -0.05 & -0.31 & -0.27 & -0.31 \\
\hline & Stein & -0.01 & -0.24 & -0.26 & -0.34 & 0.03 & -0.24 & -0.20 & -0.25 \\
\hline & BL.band & -0.01 & 0.10 & 0.45 & 0.00 & 0.21 & 0.36 & 0.62 & 0.01 \\
\hline & BL.thresh & 0.04 & 0.00 & 0.00 & 0.00 & 0.05 & 0.02 & 0.01 & 0.01 \\
\hline & IMMLE & 0.10 & 0.17 & 0.41 & 0.53 & 0.09 & 0.21 & 0.37 & 0.40 \\
\hline & IMPLE & 0.10 & 0.16 & 0.41 & 0.52 & 0.11 & 0.21 & 0.37 & 0.40 \\
\hline \multirow[t]{7}{*}{ L2 } & LW1 & 0.04 & 0.04 & 0.08 & 0.09 & -0.01 & 0.06 & 0.16 & 0.17 \\
\hline & LW2 & -0.30 & -0.99 & -2.76 & -6.32 & -0.28 & -1.41 & -4.19 & -9.47 \\
\hline & Stein & -0.23 & -0.92 & -2.69 & -6.23 & -0.13 & -1.24 & -4.01 & -9.22 \\
\hline & BL.band & -0.10 & 0.04 & 0.11 & 0.01 & 0.17 & 0.16 & 0.21 & 0.02 \\
\hline & BL.thresh & 0.06 & 0.03 & 0.01 & 0.01 & 0.20 & 0.09 & 0.04 & 0.02 \\
\hline & IMMLE & 0.07 & 0.05 & 0.10 & 0.11 & 0.06 & 0.07 & 0.15 & 0.16 \\
\hline & IMPLE & 0.07 & 0.04 & 0.09 & 0.07 & 0.15 & 0.07 & 0.17 & 0.16 \\
\hline \multirow[t]{7}{*}{ EV1 } & LW1 & -0.03 & -0.01 & 0.00 & 0.00 & -0.17 & -0.04 & 0.00 & 0.00 \\
\hline & LW2 & -0.28 & -0.61 & -1.33 & -2.49 & -0.51 & -1.00 & -2.11 & -3.42 \\
\hline & Stein & -0.24 & -0.58 & -1.31 & -2.46 & -0.41 & -0.93 & -2.05 & -3.37 \\
\hline & BL.band & -0.09 & -0.01 & 0.00 & 0.00 & -0.11 & 0.00 & 0.02 & 0.01 \\
\hline & BL.thresh & 0.01 & 0.01 & 0.01 & 0.00 & 0.08 & 0.03 & 0.02 & 0.01 \\
\hline & IMMLE & -0.01 & 0.00 & 0.00 & 0.00 & -0.05 & -0.01 & 0.00 & 0.00 \\
\hline & IMPLE & -0.01 & -0.02 & -0.01 & -0.02 & -0.07 & -0.01 & 0.00 & 0.00 \\
\hline \multirow[t]{7}{*}{ EVS } & LW1 & 0.29 & -0.27 & -0.08 & -0.23 & 0.19 & -0.79 & -0.29 & -0.43 \\
\hline & LW2 & 0.46 & 0.24 & 0.59 & 0.70 & 0.40 & 0.35 & 0.50 & 0.73 \\
\hline & Stein & 0.49 & 0.32 & 0.63 & 0.68 & 0.47 & 0.53 & 0.66 & 0.75 \\
\hline & BL.band & 0.05 & -0.12 & 0.40 & -0.01 & 0.66 & 0.56 & 0.81 & 0.00 \\
\hline & BL.thresh & 0.08 & -0.03 & -0.01 & -0.02 & 0.02 & -0.02 & 0.00 & 0.00 \\
\hline & IMMLE & 0.40 & 0.39 & 0.62 & 0.71 & 0.18 & 0.26 & 0.20 & 0.23 \\
\hline & IMPLE & 0.40 & 0.39 & 0.62 & 0.71 & 0.18 & 0.26 & 0.20 & 0.23 \\
\hline
\end{tabular}

Boldface entries correspond to the highest PRIAL for a combination of $\gamma$ and $p$ under a certain loss function. 
Table 5: PRIAL $\left(\boldsymbol{\Sigma}_{\mathbf{3}}\right)$ : Proportion Reduction in Integrated Average Loss of seven estimators compared to $S$ for various loss functions, and values for $p$ and $\gamma$.

\begin{tabular}{|c|c|c|c|c|c|c|c|c|c|}
\hline \multirow[t]{3}{*}{ Loss } & \multicolumn{2}{|l|}{$\gamma(p / N)$} & \multicolumn{3}{|c|}{0.2} & \multicolumn{4}{|c|}{0.8} \\
\hline & $\mathrm{p}$ & 10 & 20 & 50 & 100 & 10 & 20 & 50 & 100 \\
\hline & $\mathrm{n}$ & 50 & 100 & 250 & 500 & 13 & 25 & 63 & 125 \\
\hline \multirow[t]{7}{*}{$\mathrm{LS}$} & LW1 & 0.74 & 0.78 & 0.79 & 0.80 & 0.91 & 0.94 & 0.95 & 0.95 \\
\hline & LW2 & 0.74 & 0.79 & 0.81 & 0.81 & 0.88 & 0.92 & 0.95 & 0.95 \\
\hline & Stein & 0.69 & 0.75 & 0.79 & 0.80 & 0.75 & 0.82 & 0.88 & 0.91 \\
\hline & BL.band & 0.55 & 0.65 & 0.80 & 0.88 & 0.83 & 0.87 & 0.91 & 0.94 \\
\hline & BL.thresh & 0.32 & 0.56 & 0.65 & 0.66 & 0.16 & 0.37 & 0.87 & 0.92 \\
\hline & IMMLE & 0.41 & 0.45 & 0.48 & 0.49 & 0.32 & 0.35 & 0.38 & 0.38 \\
\hline & IMPLE & 0.50 & 0.53 & 0.56 & 0.57 & 0.42 & 0.45 & 0.48 & 0.48 \\
\hline \multirow[t]{7}{*}{ LQ } & LW1 & 0.72 & 0.76 & 0.78 & 0.79 & 0.86 & 0.91 & 0.92 & 0.93 \\
\hline & LW2 & 0.76 & 0.80 & 0.82 & 0.82 & 0.88 & 0.92 & 0.94 & 0.94 \\
\hline & Stein & 0.70 & 0.76 & 0.80 & 0.81 & 0.80 & 0.85 & 0.89 & 0.91 \\
\hline & BL.band & 0.54 & 0.64 & 0.79 & 0.87 & 0.76 & 0.82 & 0.87 & 0.91 \\
\hline & BL.thresh & 0.30 & 0.53 & 0.65 & 0.67 & 0.24 & 0.38 & 0.80 & 0.89 \\
\hline & IMMLE & 0.39 & 0.43 & 0.46 & 0.47 & 0.37 & 0.42 & 0.44 & 0.45 \\
\hline & IMPLE & 0.61 & 0.63 & 0.65 & 0.65 & 0.71 & 0.72 & 0.73 & 0.73 \\
\hline \multirow[t]{7}{*}{$\mathrm{LF}$} & LW1 & 0.39 & 0.41 & 0.43 & 0.43 & 0.59 & 0.63 & 0.64 & 0.64 \\
\hline & LW2 & 0.40 & 0.41 & 0.41 & 0.41 & 0.60 & 0.62 & 0.63 & 0.63 \\
\hline & Stein & 0.38 & 0.39 & 0.41 & 0.41 & 0.52 & 0.56 & 0.59 & 0.61 \\
\hline & BL.band & 0.23 & 0.32 & 0.51 & 0.62 & 0.47 & 0.53 & 0.60 & 0.68 \\
\hline & BL.thresh & 0.11 & 0.20 & 0.23 & 0.23 & 0.13 & 0.22 & 0.51 & 0.59 \\
\hline & IMMLE & 0.21 & 0.23 & 0.25 & 0.26 & 0.23 & 0.27 & 0.29 & 0.29 \\
\hline & IMPLE & 0.29 & 0.29 & 0.29 & 0.29 & 0.42 & 0.42 & 0.42 & 0.41 \\
\hline \multirow[t]{7}{*}{ L1 } & LW1 & 0.45 & 0.55 & 0.60 & 0.61 & 0.63 & 0.70 & 0.72 & 0.72 \\
\hline & LW2 & 0.44 & 0.53 & 0.57 & 0.57 & 0.62 & 0.68 & 0.73 & 0.73 \\
\hline & Stein & 0.43 & 0.51 & 0.56 & 0.56 & 0.54 & 0.60 & 0.64 & 0.63 \\
\hline & BL.band & 0.39 & 0.36 & 0.49 & 0.64 & 0.65 & 0.71 & 0.71 & 0.73 \\
\hline & BL.thresh & 0.39 & 0.46 & 0.27 & 0.17 & 0.18 & 0.36 & 0.78 & 0.74 \\
\hline & IMMLE & 0.26 & 0.34 & 0.41 & 0.44 & 0.22 & 0.26 & 0.28 & 0.28 \\
\hline & IMPLE & 0.32 & 0.38 & 0.44 & 0.46 & 0.38 & 0.38 & 0.38 & 0.37 \\
\hline \multirow[t]{7}{*}{$\mathrm{L} 2$} & LW1 & 0.57 & 0.68 & 0.78 & 0.80 & 0.82 & 0.87 & 0.89 & 0.89 \\
\hline & LW2 & 0.54 & 0.59 & 0.63 & 0.63 & 0.82 & 0.84 & 0.84 & 0.84 \\
\hline & Stein & 0.58 & 0.62 & 0.64 & 0.64 & 0.78 & 0.80 & 0.81 & 0.81 \\
\hline & BL.band & 0.53 & 0.51 & 0.70 & 0.84 & 0.87 & 0.89 & 0.90 & 0.92 \\
\hline & BL.thresh & 0.54 & 0.61 & 0.18 & -0.09 & 0.32 & 0.50 & 0.94 & 0.90 \\
\hline & IMMLE & 0.42 & 0.53 & 0.62 & 0.65 & 0.42 & 0.49 & 0.51 & 0.50 \\
\hline & IMPLE & 0.49 & 0.57 & 0.64 & 0.67 & 0.63 & 0.62 & 0.59 & 0.57 \\
\hline \multirow[t]{7}{*}{ EV1 } & LW1 & 0.01 & 0.20 & 0.49 & 0.59 & 0.55 & 0.61 & 0.70 & 0.73 \\
\hline & LW2 & -0.11 & -0.04 & 0.20 & 0.30 & 0.55 & 0.54 & 0.57 & 0.59 \\
\hline & Stein & 0.03 & 0.04 & 0.25 & 0.34 & 0.63 & 0.60 & 0.60 & 0.61 \\
\hline & BL.band & -0.12 & -0.03 & 0.42 & 0.65 & 0.73 & 0.72 & 0.76 & 0.84 \\
\hline & BL.thresh & 0.33 & -0.05 & -0.39 & -0.43 & 0.27 & 0.44 & 0.89 & 0.74 \\
\hline & IMMLE & 0.30 & 0.47 & 0.71 & 0.80 & 0.61 & 0.75 & 0.84 & 0.88 \\
\hline & IMPLE & 0.25 & 0.42 & 0.70 & 0.82 & 0.56 & 0.61 & 0.57 & 0.55 \\
\hline \multirow[t]{7}{*}{ EVS } & LW1 & 0.72 & 0.78 & 0.81 & 0.80 & 0.74 & 0.82 & 0.85 & 0.84 \\
\hline & LW2 & 0.71 & 0.81 & 0.86 & 0.87 & 0.68 & 0.78 & 0.88 & 0.90 \\
\hline & Stein & 0.64 & 0.74 & 0.84 & 0.86 & 0.46 & 0.57 & 0.69 & 0.73 \\
\hline & BL.band & 0.65 & 0.57 & 0.60 & 0.70 & 0.61 & 0.71 & 0.74 & 0.73 \\
\hline & BL.thresh & 0.43 & 0.74 & 0.76 & 0.67 & 0.09 & 0.26 & 0.75 & 0.90 \\
\hline & IMMLE & 0.36 & 0.39 & 0.39 & 0.39 & 0.12 & 0.13 & 0.12 & 0.12 \\
\hline & IMPLE & 0.33 & 0.37 & 0.38 & 0.38 & 0.12 & 0.13 & 0.12 & 0.12 \\
\hline
\end{tabular}

Boldface entries correspond to the highest PRIAL for a combination of $\gamma$ and $p$ under a certain loss function. 
Table 6: PRIAL $\left(\boldsymbol{\Sigma}_{\mathbf{4}}\right)$ : Proportion Reduction in Integrated Average Loss of seven estimators compared to $S$ for various loss functions, and values for $p$ and $\gamma$.

\begin{tabular}{|c|c|c|c|c|c|c|c|c|c|}
\hline \multirow[t]{3}{*}{ Loss } & \multicolumn{2}{|l|}{$\gamma(p / N)$} & \multicolumn{3}{|c|}{0.2} & \multicolumn{4}{|c|}{0.8} \\
\hline & $\mathrm{p}$ & 10 & 20 & 50 & 100 & 10 & 20 & 50 & 100 \\
\hline & $\mathrm{n}$ & 50 & 100 & 250 & 500 & 13 & 25 & 63 & 125 \\
\hline \multirow[t]{7}{*}{$\mathrm{LS}$} & LW1 & 0.61 & 0.81 & 0.93 & 0.96 & 0.72 & 0.87 & 0.95 & 0.97 \\
\hline & LW2 & 0.64 & 0.82 & 0.93 & 0.97 & 0.78 & 0.90 & 0.96 & 0.98 \\
\hline & Stein & 0.58 & 0.77 & 0.91 & 0.95 & 0.66 & 0.78 & 0.89 & 0.94 \\
\hline & BL.band & -1.06 & -0.01 & -0.06 & -0.08 & 0.29 & 0.17 & 0.34 & 0.03 \\
\hline & BL.thresh & -0.28 & -0.16 & 0.00 & 0.00 & 0.07 & 0.02 & -0.05 & -0.06 \\
\hline & IMMLE & 0.33 & 0.48 & 0.59 & 0.63 & 0.22 & 0.30 & 0.38 & 0.41 \\
\hline & IMPLE & 0.37 & 0.50 & 0.60 & 0.63 & 0.28 & 0.34 & 0.40 & 0.42 \\
\hline \multirow[t]{7}{*}{ LQ } & LW1 & 0.52 & 0.77 & 0.91 & 0.96 & 0.30 & 0.69 & 0.88 & 0.94 \\
\hline & LW2 & 0.65 & 0.83 & 0.93 & 0.97 & 0.75 & 0.89 & 0.96 & 0.98 \\
\hline & Stein & 0.60 & 0.78 & 0.91 & 0.96 & 0.69 & 0.81 & 0.91 & 0.95 \\
\hline & BL.band & -3.13 & 0.01 & -0.02 & -0.03 & -2.37 & -3.45 & -1.28 & 0.03 \\
\hline & BL.thresh & -0.02 & -0.02 & 0.01 & 0.00 & 0.17 & 0.09 & 0.04 & 0.02 \\
\hline & IMMLE & 0.28 & 0.46 & 0.60 & 0.65 & 0.02 & 0.27 & 0.45 & 0.52 \\
\hline & IMPLE & 0.42 & 0.54 & 0.63 & 0.71 & 0.56 & 0.64 & 0.71 & 0.73 \\
\hline \multirow[t]{7}{*}{$\mathrm{LF}$} & LW1 & 0.00 & 0.01 & 0.03 & 0.02 & 0.07 & 0.03 & 0.02 & 0.01 \\
\hline & LW2 & -0.06 & -0.23 & -0.55 & -1.08 & -0.01 & -0.32 & -0.89 & -1.65 \\
\hline & Stein & -0.04 & -0.20 & -0.54 & -1.06 & 0.03 & -0.28 & -0.85 & -1.63 \\
\hline & BL.band & -0.25 & 0.01 & 0.00 & -0.01 & -0.06 & -0.31 & -0.30 & -0.01 \\
\hline & BL.thresh & 0.00 & 0.01 & 0.00 & 0.00 & 0.06 & 0.03 & 0.01 & 0.00 \\
\hline & IMMLE & 0.01 & 0.01 & 0.02 & 0.02 & 0.04 & 0.02 & 0.02 & 0.01 \\
\hline & IMPLE & 0.02 & 0.02 & 0.02 & 0.02 & 0.10 & 0.05 & 0.03 & 0.00 \\
\hline \multirow[t]{7}{*}{ L1 } & LW1 & 0.20 & 0.39 & 0.58 & 0.69 & 0.13 & 0.34 & 0.55 & 0.67 \\
\hline & LW2 & 0.09 & 0.12 & 0.09 & 0.02 & -0.06 & -0.12 & -0.21 & -0.34 \\
\hline & Stein & 0.06 & 0.07 & 0.05 & -0.03 & -0.06 & -0.18 & -0.28 & -0.41 \\
\hline & BL.band & -0.27 & 0.00 & 0.00 & -0.01 & -0.11 & -0.03 & 0.17 & 0.02 \\
\hline & BL.thresh & 0.01 & 0.00 & 0.00 & 0.00 & 0.06 & 0.03 & 0.02 & 0.01 \\
\hline & IMMLE & 0.08 & 0.16 & 0.25 & 0.29 & -0.01 & 0.06 & 0.14 & 0.18 \\
\hline & IMPLE & 0.09 & 0.16 & 0.25 & 0.29 & 0.02 & 0.07 & 0.15 & 0.18 \\
\hline \multirow[t]{7}{*}{ L2 } & LW1 & -0.11 & -0.05 & 0.06 & 0.07 & -0.01 & -0.09 & -0.02 & -0.03 \\
\hline & LW2 & -0.44 & -1.15 & -2.81 & -6.14 & -0.43 & -1.87 & -5.05 & -11.39 \\
\hline & Stein & -0.36 & -1.07 & -2.74 & -6.05 & -0.29 & -1.69 & -4.81 & -11.11 \\
\hline & BL.band & -0.41 & 0.02 & 0.01 & 0.00 & -0.07 & -0.38 & -0.06 & -0.01 \\
\hline & BL.thresh & 0.03 & 0.02 & 0.01 & 0.00 & 0.18 & 0.08 & 0.05 & 0.01 \\
\hline & IMMLE & -0.02 & 0.01 & 0.05 & 0.05 & 0.01 & -0.02 & 0.02 & 0.01 \\
\hline & IMPLE & 0.02 & 0.01 & 0.06 & 0.05 & 0.14 & 0.02 & 0.06 & 0.00 \\
\hline \multirow[t]{7}{*}{ EV1 } & LW1 & -0.07 & -0.07 & -0.01 & -0.01 & -0.05 & -0.11 & -0.05 & -0.06 \\
\hline & LW2 & -0.31 & -0.70 & -1.39 & -2.36 & -0.46 & -1.14 & -2.18 & -3.57 \\
\hline & Stein & -0.26 & -0.66 & -1.36 & -2.33 & -0.37 & -1.05 & -2.11 & -3.51 \\
\hline & BL.band & 0.00 & 0.01 & 0.01 & 0.00 & -0.01 & -0.27 & -0.05 & -0.01 \\
\hline & BL.thresh & 0.02 & 0.01 & 0.01 & 0.00 & 0.09 & 0.04 & 0.02 & 0.00 \\
\hline & IMMLE & -0.02 & -0.02 & 0.00 & 0.00 & 0.00 & -0.03 & -0.01 & -0.02 \\
\hline & IMPLE & -0.01 & -0.04 & 0.00 & 0.00 & 0.02 & -0.03 & 0.00 & -0.02 \\
\hline \multirow[t]{7}{*}{ EVS } & LW1 & 0.80 & 0.90 & 0.97 & 0.99 & 0.72 & 0.88 & 0.95 & 0.98 \\
\hline & LW2 & 0.75 & 0.87 & 0.96 & 0.98 & 0.73 & 0.88 & 0.97 & 0.98 \\
\hline & Stein & 0.61 & 0.72 & 0.84 & 0.90 & 0.45 & 0.56 & 0.69 & 0.79 \\
\hline & BL.band & 0.09 & -0.01 & -0.02 & -0.02 & 0.56 & 0.43 & 0.40 & 0.02 \\
\hline & BL.thresh & -0.03 & -0.04 & 0.00 & 0.00 & 0.00 & -0.02 & -0.01 & -0.01 \\
\hline & IMMLE & 0.33 & 0.33 & 0.32 & 0.32 & 0.12 & 0.12 & 0.10 & 0.10 \\
\hline & IMPLE & 0.33 & 0.33 & 0.32 & 0.32 & 0.12 & 0.12 & 0.10 & 0.10 \\
\hline
\end{tabular}

Boldface entries correspond to the highest PRIAL for a combination of $\gamma$ and $p$ under a certain loss function. 
Table 7: NASDAQ Simulations: PRIAL for seven estimators under three matrix losses

\begin{tabular}{lrrr|rrr}
\hline & \multicolumn{3}{c}{$\mathrm{N}=125$} & \multicolumn{3}{c}{$\mathrm{N}=250$} \\
\hline & LS & LQ & LF & LS & LQ & LF \\
\hline LW1 & -2.04 & -123.49 & $\mathbf{0 . 0 8}$ & -1.25 & -19.26 & $\mathbf{0 . 0 5}$ \\
LW2 & -0.23 & -26.30 & -0.72 & -0.33 & -6.71 & -0.63 \\
Stein & $\mathbf{0 . 4 2}$ & 0.13 & -0.70 & $\mathbf{0 . 2 4}$ & $\mathbf{0 . 1 7}$ & -0.62 \\
BL.band & -19.15 & -4224.16 & -2.22 & 0.00 & 0.01 & 0.00 \\
BL.thresh & - & -18.51 & -2.25 & - & -33.15 & -3.55 \\
IMMLE & 0.15 & -0.28 & 0.06 & 0.11 & -0.19 & 0.03 \\
IMPLE & 0.19 & $\mathbf{0 . 3 9}$ & 0.06 & 0.13 & 0.13 & 0.04 \\
\hline
\end{tabular}

Boldface entries correspond to the highest PRIAL under a loss function. BL.thresh estimates are singular, hence $L S$ is not defined.

\section{References}

[1] G. A. Anderson. An asymptotic expansion for the distribution of the latent roots of the estimated covariance matrix. Annals of Mathematical Statistics, $36: 1153-1173,1965$.

[2] T. W. Anderson. An Introduction to Multivariate Statistical Analysis, 3rd Edition. Wiley Series in Probability and Statistics. Wiley, 2003.

[3] R. E. Barlow, D. J. Bartholomew, J. M. Bremner, and H. D. Brunk. Statistical Inference Under Order Restrictions: The Theory and Application of Isotonic Regression. Wiley Series in Probability and Mathematical Statistics. Wiley, 1972.

[4] O. E. Barndorff-Nielsen. On a formula for the conditional distribution of the maximum likelihood estimator. Biometrika, 70:343-365, 1983.

[5] P. J Bickel and E. Levina. Covariance regularization by thresholding. Annals of Statistics, 36:2577-2604, 2008.

[6] P. J Bickel and E. Levina. Regularized estimation of large covariance matrices. Annals of Statistics, 36:199-227, 2008.

[7] T. Cacoullos and I. Olkin. On the bias of functions of characteristic roots of a random matrix. Biometrika, 52:8794, 1965.

[8] D. Chételat and M. T. Wells. Improved second order estimation in the singular multivariate normal model. Journal of Multivariate Analysis, In press, 2016.

[9] D. K. Dey and C. Srinivasan. Estimation of a covariance matrix under Stein's loss. Annals of Statistics, 13:1581-1591, 1985. 
[10] L. R. Haff. The variational form of certain Bayes estimators. Annals of Statistics, 19:1163-1190, 1991.

[11] Kenneth R Hess, Keith Anderson, W Fraser Symmans, Vicente Valero, Nuhad Ibrahim, Jaime A Mejia, Daniel Booser, Richard L Theriault, Aman U Buzdar, Peter J Dempsey, et al. Pharmacogenomic predictor of sensitivity to preoperative chemotherapy with paclitaxel and fluorouracil, doxorubicin, and cyclophosphamide in breast cancer. Journal of clinical oncology, 24(26):4236-4244, 2006.

[12] A. T. James. Normal multivariate analysis and the orthogonal group. Annals of Mathematical Statistics, 25:40-75, 1954.

[13] A. T. James. The distribution of the latent roots of the covariance matrix. Annals of Mathematical Statistics, 31:151-158, 1960.

[14] W. James and C. Stein. Estimation with quadratic loss. In Proceedings of the Fourth Berkeley Symposium on Mathematical Statistics and Probability, Volume 1: Contributions to the Theory of Statistics, pages 361-379. University of California Press, 1961.

[15] D. N. Lawley. Tests of significance for the latent roots of covariance and correlation matrices. Biometrika, 43:128-136, 1956.

[16] O. Ledoit and M. Wolf. A well-conditioned estimator for large-dimensional covariance matrices. Journal of Multivariate Analysis, 88:365-411, 2004.

[17] O. Ledoit and M. Wolf. Nonlinear shrinkage estimation of large-dimensional covariance matrices. Annals of Statistics, 40:1024-1060, 2012.

[18] O. Ledoit and M. Wolf. Optimal estimation of a large-dimensional covariance matrix under Stein's loss. Technical report, Department of Economics, University of Zurich, 2014.

[19] O. Ledoit and M. Wolf. Spectrum estimation: A unified framework for covariance matrix estimation and PCA in large dimensions. Journal of Multivariate Analysis, 139:360-384, 2015.

[20] S. P. Lin and M. D. Perlman. A Monte Carlo comparison of four estimators of a covariance matrix. In P. R. Krishnaiah, editor, Multivariate Analysis VI, pages 411-429. North Holland, 1985.

[21] A. W. Marshall, I. Olkin, and B. C. Arnold. Inequalities: Theory of Majorization and Its Applications. Springer Series in Statistics. Springer, 2011.

[22] R. J. Muirhead. Latent roots and matrix variates: a review of some asymptotic results. Annals of Statistics, 6:5-33, 1978.

[23] R. J. Muirhead. Aspects of Multivariate Statistical Theory. Wiley Series in Probability and Statistics. Wiley, 2009. 
[24] N. Pal. Estimating the normal dispersion matrix and the precision matrix from a decision-theoretic point of view: a review. Statistical Papers, 34:126, 1993.

[25] F. Perron. Equivariant estimators of the covariance matrix. Canadian Journal of Statistics, 18:179-182, 1990.

[26] F. Perron. Minimax estimators of a covariance matrix. Journal of Multivariate Analysis, 43:16-28, 1992.

[27] M. Pourahmadi. Covariance estimation: The glm and regularization perspectives. Statistical Science, 26:369-387, 2011.

[28] B. Rajaratnam. Asymptotic Equivalence for Markov Chains and Eigenvalue Estimation in the Marginal Likelihood Framework. PhD thesis, Cornell University, 2006.

[29] T. Robertson, F. T. Wright, and R. Dykstra. Order Restricted Statistical Inference. Wiley Series in Probability and Mathematical Statistics. Wiley, 1988.

[30] J. B. Selliah. Estimation and testing problems in a Wishart distribution. Technical Report No. 10, Department of Statistics, Stanford University, 1964.

[31] Y. Sheena and A. Takemura. Inadmissibility of non-order-preserving orthogonally invariant estimators of the covariance matrix in the case of Stein's loss. Journal of Multivariate Analysis, 41:117-131, 1992.

[32] C. Stein. Some problems in multivariate analysis. part 1. Technical Report No. 6, Department of Statistics, Stanford University, 1956.

[33] C. Stein. Estimation of a covariance matrix. Rietz Lecture, 39th Annual Meeting, IMS, 1975.

[34] C. Stein. Lectures on the theory of estimation of many parameters. In I.A. Ibranigov and M.S. Nikulin, editors, Studies in the Statistical Theory of Estimation, volume 74 of Proceedings of Scientific Seminars of the Steklov Institute, Leningrad Division, pages 4-65, 1977.

[35] A. Takemura. An orthogonally invariant minimax estimator of the covariance matrix of a multivariate normal population. Tsukuba Journal of Mathematics, 8:367-376, 1984. 